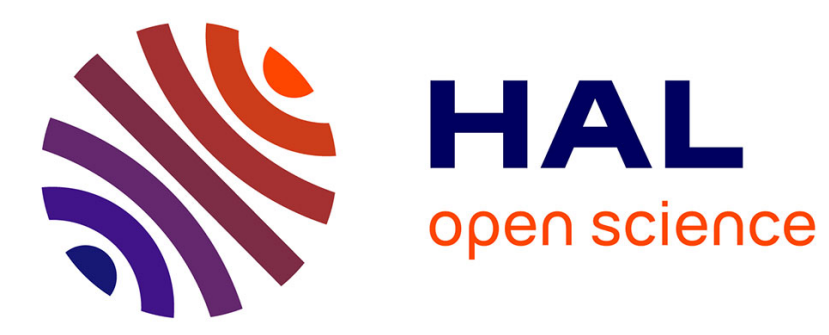

\title{
Kinetic Monte Carlo Simulations of Irradiation Effects
}

\author{
Charlotte Becquart, Normand Mousseau, Christophe Domain
}

\section{To cite this version:}

Charlotte Becquart, Normand Mousseau, Christophe Domain. Kinetic Monte Carlo Simulations of Irradiation Effects. Comprehensive Nuclear Materials 2nd edition, Volume 1, Elsevier, pp.754-778, 2020, 10.1016/B978-0-12-803581-8.11685-6 . hal-03011208v2

\section{HAL Id: hal-03011208 \\ https://hal.univ-lille.fr/hal-03011208v2}

Submitted on 14 May 2021

HAL is a multi-disciplinary open access archive for the deposit and dissemination of scientific research documents, whether they are published or not. The documents may come from teaching and research institutions in France or abroad, or from public or private research centers.
L'archive ouverte pluridisciplinaire HAL, est destinée au dépôt et à la diffusion de documents scientifiques de niveau recherche, publiés ou non, émanant des établissements d'enseignement et de recherche français ou étrangers, des laboratoires publics ou privés. 


\section{Kinetic Monte Carlo Simulations of Irradiation Effects}

\section{C.S. Becquart ${ }^{1}$, N. Mousseau ${ }^{2}$ and C. Domain ${ }^{3}$}

1 Univ. Lille, CNRS, INRA, ENSCL, UMR 8207 - UMET - Unité Matériaux et Transformations, F59000 Lille, France

2 Département de physique and Regroupement québécois sur les matériaux de pointe, Université de Montréal, Case postale 6128, succursale centre-ville, Montréal (QC) Canada H3C 3J7.

3 Département MMC, Les Renardières, F-77250 Moret sur Loing, France

Email: charlotte.becquart@univ-lille.fr

AKMC: atomic kinetic Monte Carlo

BKL: Bortz, Kalos, Lebowitz algorithm

DFT: Density Functional theory

dpa: displacement per atom

EKMC: event kinetic Monte Carlo

ER: electrical resistivity

FP: Frenkel pair

GB: grain boundaries

KMC: kinetic Monte Carlo

MC: Monte Carlo

MD: Molecular dynamics

MFRT: mean field rate theory

OKMC: object kinetic Monte Carlo

PKA: primary knocked-on atom

RIS: radiation induced segregation

RTA: residence time algorithm

SIA: self interstitial atom

TST: transition state theory

\section{Introduction}

Monte Carlo (MC) techniques are among the most used techniques for modeling materials at the atomic scale, with those of molecular dynamics. The name MC comes from the fact that this method, derived from statistical physics, uses random numbers similar to those involved in the game of roulette. The techniques of MC were born with the development of the hydrogen bomb, after the second world war. At that time, it was possible to simulate only a few hundred atoms (Wood and Parker 1957). Today simulations of several million atoms and several billion steps are quite common. Two main types of algorithms have been generated. The classic algorithm of MC developed by Metropolis (Metropolis et al. 1953) focuses on sampling the configuration space in order to construct statistical ensemble properties of the system. Focusing on equilibrium, MC allows the use of a rich variety of moves and 
importance sampling distributions to sample complex landscape, with the caveat that time is not directly accessible in the standard approach. The Kinetic Monte Carlo family of algorithms lifts this problem, when the microscopic mechanism (s) leading to a change of configuration are known, and introduces the time at the cost, of course, of limiting the sampling efficiency. The latter method is, however, essential to deal with problems of diffusion and transport in solids and is the topic of this chapter.

\section{Method}

The Monte Carlo method was originally developed by von Neumann, Ulam and Metropolis to study the diffusion of neutrons in fissionable material on the Manhattan Project and first applied to simulating radiation damage of metals more than 50 years ago by Besco (Besco 1967), Doran (Doran 1970) and later Heinisch and co-workers (Heinisch 1983).

Monte Carlo is a stochastic process and utilizes random numbers to select event probabilities from probability distributions and generate atomic configurations (Allen and Tildesley 2017), rather than the deterministic manner of molecular dynamics simulations, but remains as statistically exact, and often more efficient, when computing ensemble, or in the case of KMC methods, dynamic properties. While different Monte Carlo applications are used in computational materials science, we shall focus our attention on kinetic Monte Carlo (KMC) simulation as applied to the study of radiation damage.

The KMC methods used in radiation damage studies represent a subset of MC methods that can be classified as rejection-free, in contrast with the more classical MC methods based on the Metropolis algorithm (Metropolis and Ulam 1949) (Metropolis et al. 1953). They provide a solution to the Master Equation which describes a physical system whose evolution is governed by a known set of transition rates between possible states (Fichthorn and Weinberg 1991). The solution proceeds by choosing randomly among the various possible transitions and accepting them on the basis of probabilities determined from the corresponding transition rates. These probabilities are calculated for physical transition mechanisms as Boltzmann factor frequencies, and the events take place according to their probabilities leading to an evolution of the microstructure. The main ingredients of such models are thus a set of objects (which can resolve to the atomic scale as atoms, or point defects) and a set of reactions or (rules) that describe the manner in which these objects undergo diffusion, emission, reactions, and their rates of occurrence.

Many of the KMC techniques are based on the residence time algorithm (RTA) derived fifty years ago by Young and Elcock (Young and Elcock 1966) to model vacancy diffusion in ordered alloys. Its basic recipe involves the following: for a system in a given state, instead of making a number of unsuccessful attempts to perform a transition to reach another state, as in the case of the Metropolis algorithm (Metropolis and Ulam 1949) (Metropolis et al. 1953), the average time the system remains in its state is calculated. A transition to a different state is then performed based on the relative weights determined amongst all possible transitions, which also determines the time increment associated with the selected transition. According to standard transition state theory (see for instance (Vineyard 1957)) the frequency $\Gamma_{x}$ of a thermally activated event $x$, such as a vacancy jump in an alloy or the jump of a void can be expressed as:

$$
\Gamma_{X}=v_{X} \exp \left(-\frac{E_{a}}{k_{B} T}\right)
$$


where $v_{X}$ is the attempt frequency, $k_{B}$ is Boltzmann's constant, $T$ is the absolute temperature and $E_{a}$ is the activation energy of the jump.

During the course of a KMC simulation, the probabilities of all possible transitions are calculated and one event is chosen at each time-step, by extracting a random number, and comparing to the relative probability. The associated time-step length, $\delta t$, and average time-step length, $\Delta t$, are given by:

$$
\delta t=\frac{-\ln r}{\sum_{x} \Gamma_{x}} ; \text { and } \quad \Delta t=\frac{1}{\sum_{x} \Gamma_{X}}
$$

where $r$ is a random number between 0 and 1 . The residence time algorithm is also known as the BKL (Bortz, Kalos, Lebowitz) algorithm (Bortz et al. 1975). Other techniques are possible, as described by Chatterjee and Vlachos in Ref. (Chatterjee and Vlachos 2007).

The basic steps in a KMC simulation can thus be summarized as:

1. Calculate the probability (rate) for a given event to occur.

2. Sum the probabilities of all events to obtain a cumulative distribution function.

3. Generate a random number to select an event from all possible events.

4. Increment the simulation time based on the inverse sum of the rates of all possible events (equation 2).

5. Perform the selected event and all spontaneous events as a result of the event performed.

6. Repeat steps 1 through 4 until the desired simulation condition is reached.

Kinetic Monte Carlo models are now widely used for simulating radiation effects on materials as we will demonstrate in this chapter. Advantages of KMC models include the ability to capture spatial correlations in a full three-dimensional simulation with atomic resolution, while ignoring the atomic vibration time scales captured by molecular dynamics models, as well as the possibility to work on multiple scales. Indeed, with KMC, individual point defects, point defect clusters, solutes and impurities can be treated as objects, either on or off an underlying crystallographic lattice, as the evolution of these objects is modeled over time. Two general approaches have been used in KMC simulations, event KMC (Lanore 1974) (Dalla Torre et al. 2005) (Jourdan et al. 2011) and object KMC, which differs in the treatment of time scales or step between individual events. Within the object KMC designation, it is also possible to further sub-divide the techniques into those that explicitly treat atoms and atomic interactions, which is often denoted as atomic Kinetic Monte Carlo (AKMC), or Lattice KMC (LKMC), and which was reviewed by Becquart and Domain (Becquart and Domain 2010), versus those that track the defects on a lattice, but without complete resolution of the atomic arrangement. This later technique is predominately referred to as object Monte Carlo (OKMC), and used in such codes as BigMac (Johnson et al. 1998) (Caturla et al. 2016), LAKIMOCA (Domain et al. 2004), MMonca (MartinBragado et al. 2013) and others (Figure 1). In this chapter, we will designate by OKMC, the mesoscopic version of the object kinetic MC and by AKMC the atomistic version of the object kinetic MC. AKMC simulations are more precise but, as a consequence, as a more time consuming. The basic problem is, for this approach, to give a realistic description of all the relevant atomic displacement mechanisms: the displacements directly resulting from radiation damage, ballistic mixing as well as the thermally activated jumps of point defects, the point defect elimination mechanisms, including the way the point defect or solute migration barriers depend on the local atomic environment. In OKMC (the object version of it) and EKMC simulations, the issue is to determine the relevant mechanisms that can be activated as well as the effective parameters, usually obtained from atomic models. 


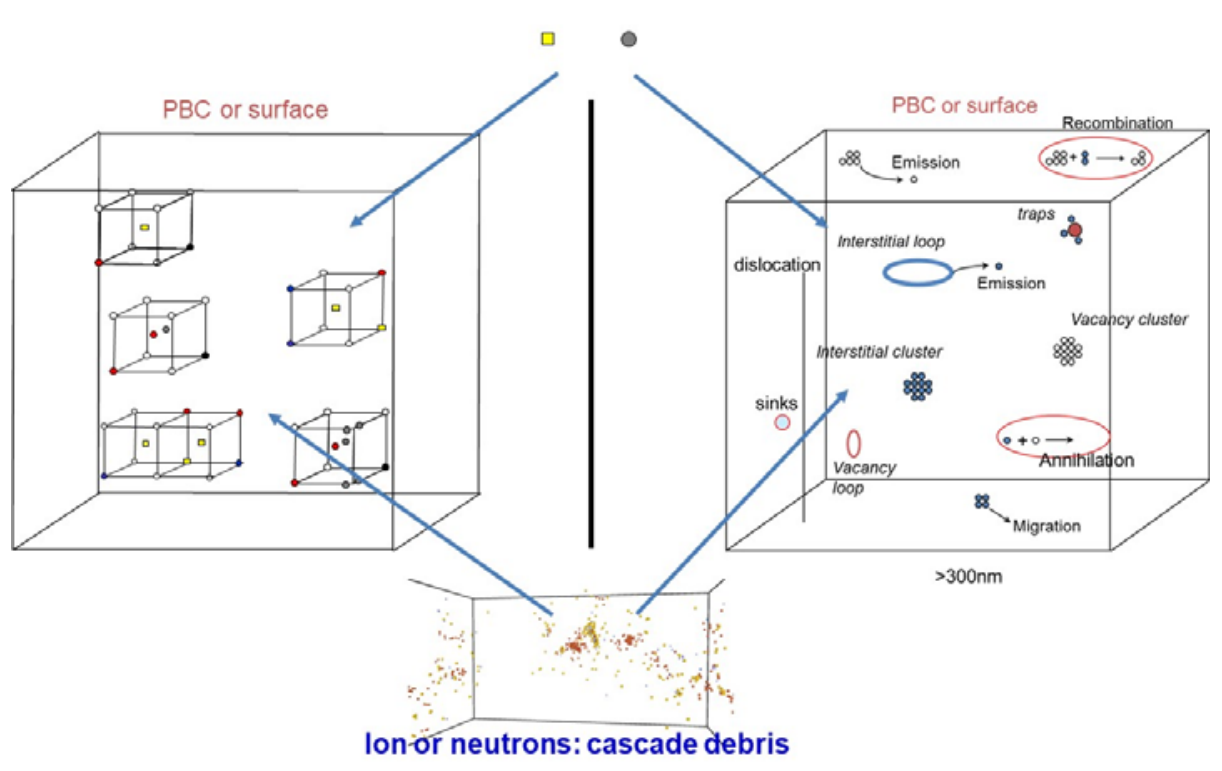

Figure 1: AKMC supercell (left) vs OKMC supercell (right). The AKMC considers atoms and point defects whereas the OKMC considers objects. In the AKMC a 5 vacancy cluster is made of 5 vacancies that behave independently from one another, whereas in the OKMC a 5 vacancy cluster is a single object. In both approaches, the irradiation consists in the introduction of point defects, isolated or in clusters depending on the irradiation type [chapter 00020].

As KMC modeling of radiation damage involves tracking the location and fate of all defects, impurities and solutes as a function of time to predict microstructural evolution, the starting point in these simulations is often the primary damage state, i.e. the spatially correlated locations of vacancies, selfinterstitials and transmutants produced in displacement cascades (Becquart et al. 2018a) (Nordlund et al. 2018) resulting from irradiation and obtained from MD [chapter 00018 ] or BCA [chapter 00028] simulations, along with the displacement or damage rate which sets the time scale for defect introduction. The rates of all reaction - diffusion events then control the subsequent evolution or progression in time, and are determined from appropriate activation energies for diffusion and dissociation, as well as the reactions that occur between species are key input, which, as stated previously, are assumed to be known. The defects execute random diffusion jumps (in one, two or three dimensions depending on the nature of the defect) with a probability (rate) proportional to their diffusivity. Similarly, cluster dissociation rates are governed by a dissociation probability that is controlled by the binding energy of a particle to the cluster. The events to be performed and associated time step for each Monte Carlo sweep are chosen from the RTA (Young and Elcock 1966) (Bortz et al. 1975). The list of possible events can be either predetermined or calculated on-the-fly as will be discussed in the next section. Note that in the EKMC approach (Lanore 1974) (Dalla Torre et al. 2005) (Jourdan et al. 2011), the microstructure also consists of objects. The crystal lattice is ignored and objects' coordinates can change continuously. The only events considered are those which lead to a change in the defect population, namely: clustering of objects, emission of mobile species, elimination of objects on fixed sinks (surface, dislocation), or the annihilation of objects on their anti-defect. The migration of an object in its own right is considered an event only if it ends up with a reaction which 
changes the defect population. In this case the migration step and the reaction are processed as a single event; otherwise, the migration is performed only once at the end of the EKMC time interval $\Delta t$. In contrast to the residence time algorithm, where all rates are lumped into one total rate to obtain the time increment, in an EKMC scheme the time delays of all possible events are calculated separately and sorted by increasing order in a list. The event corresponding to the shortest delay, $\tau_{s}$, is processed first, and the remaining list of delay times for other events is modified accordingly by eliminating the delay time associated with the particle that just disappeared, adding delay times for a new mobile object, etc. A comparison between EKMC and OKMC can be found in (Barbu et al. 2005) (Domain and Becquart 2019).

\section{II-1 classical approach: predetermined event list}

The traditional approach to KMC has been to build a list of predetermined events with their associated activation energy. For the diffusion events, the activation energies are the migration energies (and jump frequencies) of the moving species: point defects, point defect clusters, transmutants or solute atoms as well as Foreign Interstitial Atoms (FIA) such as carbon, helium and hydrogen, isolated or in clusters with or without point defects. When the local chemical environment is complex, i.e. when the system is composed of several atomic elements, these data are not straightforward to evaluate and often associated with this approach has been the use of rigid lattices. The rigid lattice approximation allows a fast estimation of the activation barriers through simple heuristic approaches such as the kinetically resolved activation (KRA), according to Van der Ven (Van der Ven et al. 2001), or final initial state energy (FISE) approximation, according to the terminology adopted by Vincent et al. (Vincent et al. 2008b), based on the Kang and Weinberg decomposition of migration energy barriers (Kang and Weinberg 1989). Such simple models include broken bonds or cut bond models (Soisson et al. 1996) as well as models based on cluster expansions of the Hamiltonian (Sanchez et al. 1984), the simplest ones being pair models (Liu et al. 1997) (Vincent et al. 2008a) (Ngayam-Happy et al. 2010). When interatomic potentials are available for the material modeled, then clever techniques such as artificial neural network (ANN) (Domingos et al. 2006) (Djurabekova et al. 2010) (Castin and Malerba 2010) can also be used. Interatomic potentials have limitations, however, and are only available at the moment, often with limited precision, for simple materials such as binary or ternary alloys [chapter 00019]. Nevertheless, interatomic potentials are often essential as they allow to get rid of the rigid lattice constrain as the activation barriers can be determined no matter the positions of the atoms around the moving species.

\section{II-2 On-the fly kMCs}

Restriction to lattices positions, when evolving the system, greatly limits the applications of KMC but is imposed for technical reasons. Diffusion mechanisms can become rapidly complex and difficult to predict in distorted and irregular environments. They are, moreover, generally difficult to classify, especially before the launch of a simulation, greatly limiting the capacity to construct a relevant event catalog.

Overcoming these limitations is challenging and computational costly. The first off-lattice KMC simulation was performed by Henkelman and Jónsson (Henkelman and Jónsson 2001) looking at Al on $\mathrm{Al}$ growth. Without catalog, the simulation generated an event list at every step, using 25 open-ended saddle searches generated with the dimer method. While the number of searches was too small to ensure that all events had been found, this work still identified new extended low-barrier events that modified considerably the kinetics of growth. Over the following years, a number of approaches were introduced to reduce the computational cost of off-lattice KMC simulations by introducing a cataloging approach. 
While still lattice-based, Trushin et al (Trushin et al. 2005) introduced an algorithm where the catalog was adjusted on-the-fly as the system evolved, allowing the study of more complex environments and, more important, the inclusion, in the barrier calculation, of a larger area, going beyond the standard first and second neighbor shells.

The first off-lattice KMC algorithm with on-the-fly cataloging was introduced by El Mellouhi et al. (ElMellouhi et al. 2008). Coupling an open-search method, the activation-relaxation technique (ART nouveau) (Barkema and Mousseau 1996) (Malek and Mousseau 2000) with a topological classification, NAUTY (McKay 1981) (McKay and Piperno 2014), the kinetic Activation-Relaxation Technique (kART) associates local graphs with a unique event list, that is generated as new topologies are identified. By removing the need for an underlying crystalline lattice, k-ART allows the creation of event catalogs for any environment even fully disordered (Béland et al. 2011) (Béland et al. 2013) (Joly et al. 2012) (Béland et al 2012). These generic cataloged events provide the basis for computing the specific energy barrier associated with each relevant event, as its exact geometry is reconstructed and exactly converged to the real saddle point. Such effort is costly, of course, even with the creation of an event catalog that reduces significantly the time spent for searching for events. This additional expense is well worth in many cases, even for apparently simple systems as demonstrated by the unexpectedly rich event catalog generated by k-ART for point defects (vacancies and interstitials) in crystalline Fe (Brommer et al. 2014). Vacancy diffusion and clustering processes in body-centered-cubic (bcc) Fe were studied using the kinetic activation-relaxation technique (k-ART), an off-lattice kinetic Monte Carlo method with onthe-fly catalog building capabilities (Brommer et al. 2014). For monovacancies and divacancies, k-ART recovered previously published results while clustering in a 50-vacancy simulation box agreed with experimental estimates. Applying k-ART to the study of clustering pathways for systems containing from one to six vacancies, allowed to find a rich set of diffusion mechanisms. In particular, it was shown that the path followed to reach a hexavacancy cluster influences greatly the associated mean-square displacement. Aggregation in a 50-vacancy box also showed a notable dispersion in relaxation time associated with effective barriers varying from 0.84 to $1.1 \mathrm{eV}$ depending on the exact pathway selected. The effects of long-range elastic interactions between defects were isolated by comparing to simulations where those effects were deliberately suppressed. This allowed to demonstrate that in bcc $\mathrm{Fe}$, suppressing long-range interactions mainly influences kinetics in the first $0.3 \mathrm{~ms}$, slowing down quick energy release cascades seen more frequently in full simulations, whereas long-term behavior and final state are not significantly affected (Brommer et al. 2014). Similar studies were performed in Si (Trochet et al. 2015) and $\mathrm{Ni}$ (Mahmoud et al. 2018), with the identification of previously unreported multistep diffusion mechanisms that are as fast or even faster than those used generally. For example, while the barrier for the single Ni self interstitial is only $0.15 \mathrm{eV}$, Ni tetra-self-interstitials (Figure 2) diffuse with almost zero energy barrier $(0.004 \mathrm{eV})$ through a three-step process involving a rotation and a jump mechanisms (Mahmoud et al. 2018). 
Interstitial atoms

a)

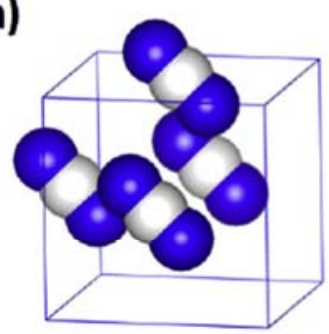

4SIA-GS

b)

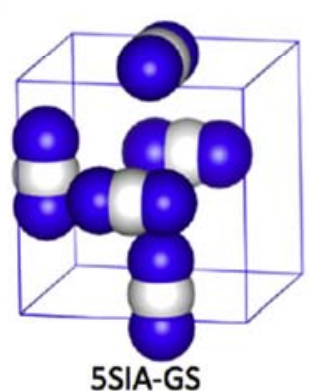

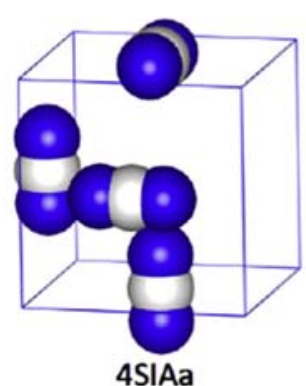

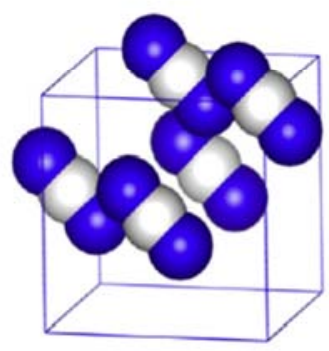

5SIAa
Vacancies

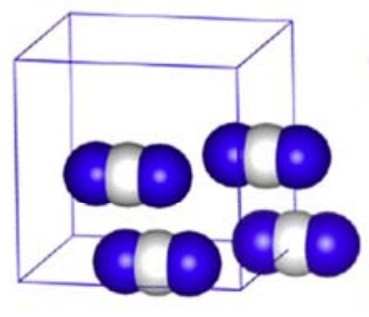

4 SIAb

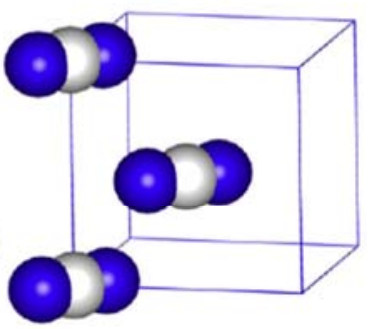

$4 S I A C$

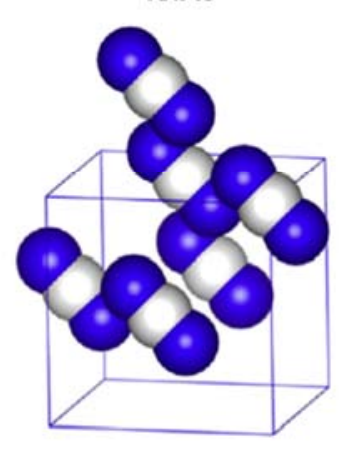

SSIAC

Figure 2: Schematic illustration of lowest-energy states for one to five self-defects in nickel using an EAM potential, these ground states were generated through KMC evolution using k-ART, allowing to take into account full elastic deformations. Starting from a random distribution of interstitials, the kinetic evolution of each 4000 + interstitial atoms system is allowed to proceed, unbiased, at $500 \mathrm{~K}$. 1) Ground states for vacancies and 2) self-interstitials. a), b), c), d) and e) present the structure with one, two, three, four and five mono-self-defects for each case. From (Mahmoud 2018)

Off-lattice KMC methods are even more important when strain and randomness play an important role, such as for characterizing the $\langle 100\rangle$-interstitial loop formation in $\mathrm{Fe}$ (Xu et al. 2013), following the relaxation of ion-bombarded silicon over long-time (more than $1 \mathrm{~s}$ ) (Béland et al. 2013) (Béland et al 2013) or understanding $C$ diffusion at an Fe grain boundary, where pathways are strongly influenced by the local deformation (Restrepo et al. 2018).

Over the last decade, a number of other off-lattice or near-off lattice approaches have been proposed, with or without a catalog. Xu and collaborators (Xu et al. 2011) for example, proposed the self-evolving atomistic kinetic Monte Carlo (SEAKMC), which defines active volume over which activated mechanisms are searched. As discussed recently (Béland et al. 2015a), while SEAKMC and k-ART produce similar results, the first method is faster in simpler environments, such as the recombination of a Frenkel pair (Nakashima et al. 2015) while the second one is more flexible for handling complex materials.

All KMC methods, including those off-lattice, are only as good as their event catalog and their evaluations of the respective rates. While open-ended search algorithms, such as ART nouveau, provide extensive catalogs (Malek and Mousseau 2000), none can guarantee completeness. To alleviate this limitation, (Chill and Henkelman 2014) introduced a coupling with high temperature molecular dynamics to obtain a better evaluation of the error on the catalog. This analysis is valid as a long as the harmonic approximation of transition state theory is valid, i.e., as long as attempt frequencies are not affected by the temperature, which is the standard approximation used in KMC approaches. Yet, recent 
results show that this approximation is not valid when the barrier energy is comparable to the temperature (Swinburne and Marinica 2018). This suggests that in the presence of a wide barrier distribution: (i) the harmonic approximation does not provide the correct rates and (ii) it is not possible to rely on high-temperature MD to evaluate low-temperature kinetics.

Off-lattice adaptive KMC methods are opening the application of accelerated methods to more complex environments and systems, providing precise and detailed information as to the dynamics of complex materials. At the same time, these applications are forcing a reevaluation of standard approximations, such as the harmonic approximation in transition state theory (TST), and the use of relatively simple force fields in non-crystalline and multicomponent environments. We can expect considerable developments over the coming decade to widen the scope of application of these methods along with new insights over questions that had been long neglected by lack of access to the appropriate time and length scales. At the moment, however, given their cost, compared with lattice-based KMC methods, off-lattice approaches, such as k-ART, are best used for specific problems. First, disordered environments, where no other method can be applied. Second, systems where the possible number of environments is unknown or too large to systematically explore before hand. At the moment, however, while active development is going on regarding this issues and solutions could be introduced in the near future, the handling of complex ensemble of atoms/defects moving together (such as handled in object $\mathrm{KMC}$ ), large moving defects (e.g. dislocations) and parallel evolution of a number of independent structures is limited.

Note that rigid lattices are not mandatory either for larger scale KMC approaches such as OKMC and EKMC, as demonstrated for instance in the codes BIGMAC (Caturla et al. 2016) and JERK (Dalla Torre et al. 2005).

On-the-fly off lattice KMC is one of the methods that allow to go beyond the reaches of classical MD along with other long time scale atomic methods such as various accelerated MD methods (Voter et al. 2002) (Uberuaga and Perez 2018) (Henkelman et al. 2018) [chapter 00018].Choosing between these MD methods and off-lattice KMC approaches, which are much heavier, computationally speaking, than lattice-based, is, therefore, not automatic. While each problem is specific and methods evolve rapidly, as a general rule, accelerated MD methods are efficient when the energy distribution of the relevant barriers is narrow and relatively well separated from the other mechanisms in the simulation. Off-lattice $\mathrm{KMC}$ are better at handling flickers and treating wider energy barrier distributions.

Now that the basis of the KMC methods have been introduced, we present the specific issues pertinent to the understanding of radiation damage and how they are / can be implemented using KMC techniques.

\section{Radiation damage specific issues}

The KMC technique has been applied to a large range of materials science phenomena such as film deposition (Martinez-Martinez et al. 2018), corrosion (Herbert et al. 2014) (Fujii et al. 2015), precipitation (Becquart and Soisson 2018). Each phenomenon needs to be described in its own way and the next section presents the peculiarity of radiation damage modelling. For a description of radiation effects on microstructures see for instance chapter 00003.

\section{III-1 Irradiation (introduction of cascades, FP)}

As mentioned in the introduction and chapter 00003, the introduction of high energy particles (neutrons, ions...) will create a damage that depends, among other factors, on the flux of these particles. The "irradiation" rate, i.e. the rate of impinging particles is usually transformed into a production rate (number per unit time and volume) of randomly distributed displacement cascades of different energies $(5,10,20, \ldots \mathrm{keV})$, as well as residual Frenkel Pairs (FP). New cascade debris are then injected randomly 
in the simulation box, at the corresponding rate. The cascade debris can be obtained by MD simulations for different recoil energies $\mathrm{T}$, or introduced based on the number of FP expected from displacement damage theory. In the case of KMC simulation of electron irradiation, FPs are introduced randomly in the simulation box according to a certain dose rate, assuming most of the time that each electron is responsible for the formation of only one FP. This assumption is valid for electrons with energies close to $1 \mathrm{MeV}$ (much lower energy electrons may not produce any FP, whereas higher energy ones may produce small displacement cascades with the formation of several vacancies and self interstitial atoms (SIAs)).

The dose is updated by adding the incremental dose associated with the scattering event of recoil energy T, using the Norgett-Robinson-Torrens expression (Norgett et al. 1975) for the number of displaced atoms. In this model, the accumulated displacement per atom (dpa) [chapter 00020] is given by:

$$
\text { displacement per subcascade }=0.8 T / 2 E_{D}
$$

where $\mathrm{T}$ is the damage energy, i.e. the fraction of the energy of the particle transmitted to the Primary Knocked-on Atom (PKA) [chapter 00020] as kinetic energy and $E_{D}$ is the threshold displacement energy (e.g. $40 \mathrm{eV}$ for Fe and Reactor Pressure Vessels (RPV) steels (ASTM E693 1994)).

The production rate (the number and energy of the PKAs, i.e. the PKA spectrum) are typically obtained from codes based on the Binary Collision Approximations (BCA) such as SRIM (Ziegler et al. 2010) or Marlowe (Robinson 1989) but other approximations and codes are available [chapter 00028]. In the case of ion implantation, one further needs to know the ion as well as the point defect implantation profiles. Long term simulations of accumulation of cascades have been modeled using KMC techniques (Becquart et al. 2005) (Caturla et al. 2006) (Souidi et al. 2006) (Becquart et al. 2006) (Souidi et al. 2011) (Valles et al. 2015). They are ways to investigate the sensitivity of the system to the properties of the displacement cascades (their spatial extent, morphology and the spatial correlation of defects) in the evolution of point defect cluster size distributions. It has been shown that there is no simple answer and the sensitivity can be little or strongly dependent on other parameters such as the mobility of defects, the type of interactions, the presence of impurities and the temperature (Becquart et al. 2006) (Souidi et al. 2011) (Valles et al. 2015). Additional work is thus needed to obtain a more reliable estimate of the primary damage, for the full high energy particle (neutrons, Fe and to a lesser extent electrons) spectra in particular in alloys.

\section{III-2 Point defect fluxes}

As mentioned previously, one of the consequence of irradiation is the formation of a large amount of point defects of both types: vacancies and SIAs that will migrate in the material, creating fluxes of point defects as well as solute atoms. It was shown in many cases that the interaction between the point defect distribution, the driving force for solute segregation and precipitation when solubility limits are exceeded, will lead to complex microstructural evolutions, which depend on the atomic-scale diffusion properties used in the models (Soisson 2006). Usually the rates of diffusion can be obtained from the knowledge of the migration barriers. These, or at least the most important, must then be known for all the diffusing "objects"; i.e. for the point defects in AKMC, OKMC and EKMC, or the clusters in OKMC or EKMC. For isolated point defects, the migration barriers can be obtained from experimental data, i.e. from diffusion coefficients, or theoretically, using the different approaches described in section II.1, or directly from either ab initio calculations as described for instance in (Caturla et al. 2008) (Becquart and Domain 2009) or MD simulations as described in (Soneda and Rubia 1998). Permanent fluxes of point defects towards the sinks are thus sustained by irradiation: the flux of SIAs corresponding to a flux of atoms towards the sinks, whereas the flux of vacancy corresponds to a flux of atoms away from the sinks. These fluxes will lead to microstructural changes such as precipitation or segregation as described 
in (Becquart and Soisson 2018) and in more details in [chapter 00008]. It is thus important to model correctly the diffusion properties of the moving species as well as the "trapping" properties of the different sinks: free surfaces, grain boundaries, dislocations... or traps. A typical example is the impact of point defect diffusion on $\mathrm{Zr}$ growth as shown in (Samolyuk et al. 2014).

\section{III-3 Formation and migration of SIAs and SIA clusters / loops}

Irradiation will lead to the disruption of the crystallinity and the formation of numerous point defects which would not be present in such large amounts in the material under equilibrium conditions. This is particularly the case of SIAs which are not found in metals under thermal equilibrium because of their large formation energies (around $4 \mathrm{eV}$ for $\mathrm{Fe}, 10 \mathrm{eV}$ for $\mathrm{W}$ ) and their very low migration barriers (around $0.3 \mathrm{eV}$ in $\mathrm{Fe}$, and close to 0 in $\mathrm{W}$ ). SIAs move very quickly and tend to form clusters in the form of dislocations loops: dislocation loops of interstitial type have been observed in all kinds of alloys: RPV steels, model alloys ( $\mathrm{FeCu}, \mathrm{FeMnNi}, \mathrm{FeCuNiMn}), \mathrm{Fe}, \mathrm{W}$ and $\mathrm{W}$ alloys ...(Hernández-Mayoral and Gómez-Briceño 2010) (Meslin et al. 2008) (Meslin et al. 2010) (Yi et al. 2015). One issue regarding SIA loops is their burgers vector. For instance, in Fe, two kinds of loops are observed: $1 / 2\langle 111\rangle$ loops which move very easily by one dimensional glide (Bacon et al. 2009) specially at low temperatures (Arakawa et al. 2007) and $\langle 100\rangle$ loops which are sessile. It is thus important to, on the one hand, include in the KMC models the correct amount of each family of loops and, on the other hand, model correctly their mobility or lack of. Indeed, when they are mobile, SIA loops move very quickly in a 1D motion. This has an impact on their sink strength, i.e. their capacity to interact/attract other point defects and solute atoms. This has been demonstrated in various cases using KMC (Heinisch et al. 1999) (Heinisch et al. 2000) (Ghoniem et al. 2002) (Wen et al. 2009) (Amino et al. 2011) (Jansson et al. 2013). Furthermore, the interaction of these loops with other loops or solute atoms can lead to changes in the loop Burgers vector and thus in their mobility. This has been recently a scope for on-the-fly KMC simulations (Xu et al. 2013) (Béland et al. 2015b) and needs to be accounted for in modelling microstructure evolution under irradiation. Another issue related to the formation and the mobility of SIA loops is that they interact with solute atoms (Domain and Becquart 2018). KMC studies have shown that solute atoms can confine SIA loop diffusion to short segments (Hudson et al. 2004), slow down the loops as proposed in (Chiapetto et al. 2015) and change the loop saturation density (Terentyev and Martin-Bragado 2015). It is thus necessary to implement these effects correctly, either by introducing the solutes explicitly in the simulation as in (Hudson et al. 2004) (Lee et al. 2009) (Gámez et al. 2011) (Terentyev and Martin-Bragado 2015) or implicitly by changing the diffusion properties of the loops to reflect the alloying content as in (Chiapetto et al. 2015) and (Bonny et al. 2018). Another complication with SIA clusters and loops is the fact that the strain they generate in the lattice is not negligible and should be accounted for. KMC simulations have indeed shown that elastic interaction between defects impact on point defect recombination and the formation of clusters (Hudson et al. 2005), the trapping of SIA clusters (Wen et al. 2009) or that of dislocation loops (Mason et al. 2014). Another demonstration of the importance of elastic interactions can be found in (Wen et al. 2005). These authors showed that elastic interactions help SIAs rotate and organize themselves into rafts or dislocation decorations. Note that impurities and solute atoms also interact with vacancy clusters and loops (Messina et al. 2015) but this has not been so far the object of many KMC simulations (Chiapetto et al. 2015).

\section{III-4 Ballistic mixing}

Ballistic mixing refers to the athermal displacements of atoms within displacement cascades. It always tends to disorder the alloys, irrespective of their equilibrium properties and can lead to precipitate or nanoparticle dissolution (Krasnochtchekov et al. 2007a) (Krasnochtchekov et al. 2007b) (Soisson et al. 2018), (Vu et al. 2016). It has been proposed for instance as a reason for the lack of $\alpha$ ' precipitation in $\mathrm{FeCr}$ alloys irradiated under high ion flux when the concentration of sinks is high enough (Soisson et al. 
2018). Ballistic mixing is thus an irradiation effect competing with the acceleration of diffusion due to the introduction of point defect sursaturations. A ballistic diffusion coefficient can be defined, that depends on the dose and the number of replacement per displacement (Martin and Bellon 1996). KMC simulations have shown that these competing effects can lead to complex microstructures: different stages of order (Ye and Bellon 2004), the roughening of interfaces, self-organization of precipitates at a mesoscale (Bellon and Enrique 2001), (Bellon and Averback 2015), patterning (Lear et al. 2017).

\section{III-5 Transmutation}

The rate of producing transmutations can also be included in KMC models, as determined from the reaction rate density determined from the product of the neutron cross section and neutron flux. Like the irradiation rate, the volumetric production rate is used to introduce an appropriate number of transmutants, such as helium that is produced by $(n, \alpha)$ reactions in the fusion neutron environment, where the species are introduced at random locations within the material. In $\mathrm{W}$, transmutation will for instance lead to the formation of Re and Os in non negligible amounts (Gilbert and Sublet 2011). These elements have a strong impact on the mobility of SIAs (Suzudo et al. 2015) (Huang et al. 2017) and can lead to the formation of brittle intermetallic phases.

The next section is devoted to present typical studies pertaining to radiation damage undertaken using the KMC approach.

\section{IV kMC studies related to radiation damage}

\section{IV-1 Annealing of cascades and source term determination}

A large number of KMC simulations has been dedicated to the investigation of cascade annealing either to study the cascade efficiency of defects from their nascent cascades (i.e. how many point defect survive intracascade recombination and will feed the point defect fluxes) as in (Diaz et al. 1997) (Soneda and Rubia 1998) (Gao et al. 1999) (Alonso et al. 2000) (Domain et al. 2004) (Pelaz et al. 2007) (Kittiratanawasin et al. 2009) (Yoshiie et al. 2011) (Suzudo et al. 2012) (Xu et al. 2012b)(Xiao et al. 2016) (Wu et al. 2017) or to produce the source term for other mesoscale codes such as rate equation cluster dynamics (Adjanor et al. 2010) (Jourdan and Crocombette 2012) (Jourdan and Crocombette 2018). These studies have shown that the relative mobilities of point defects and point defect clusters as well as the migration mechanisms (Figures 3 and 4) have a crucial impact on the cascade efficiency and the annealing of the cascades (Domain et al. 2004) (Xu et al. 2012b) (Nandipati et al. 2015a) (Nandipati et al. 2015b). Depending on the material, the most mobile defect is the SIA or the vacancy, and it was shown that the relative point defect clustering trends versus temperature in addition to the disparate mobilities of SIAs versus vacancies, changes the annealing efficiency i.e. (the ratio of the number of defects after and before annealing). In W, it exhibits an inverse U-shape curve as a function of temperature (Nandipati et al. 2015a). In some very specific cases, it is possible to neglect some of the point defect reactions thus speeding up the calculations as was shown by (Pelaz et al. 2007) in ion implanted $\mathrm{Si}$, but in general one must account explicitly for both point defect populations. Furthermore, the very recent study by Jourdan et al. (Jourdan and Crocombette 2018) shows that the cascade annealing depends on the point defect concentrations and must thus be evaluated on-the-fly or for different point defect concentrations unlike it has been typically done. 
(a) SET I, SIA clusters
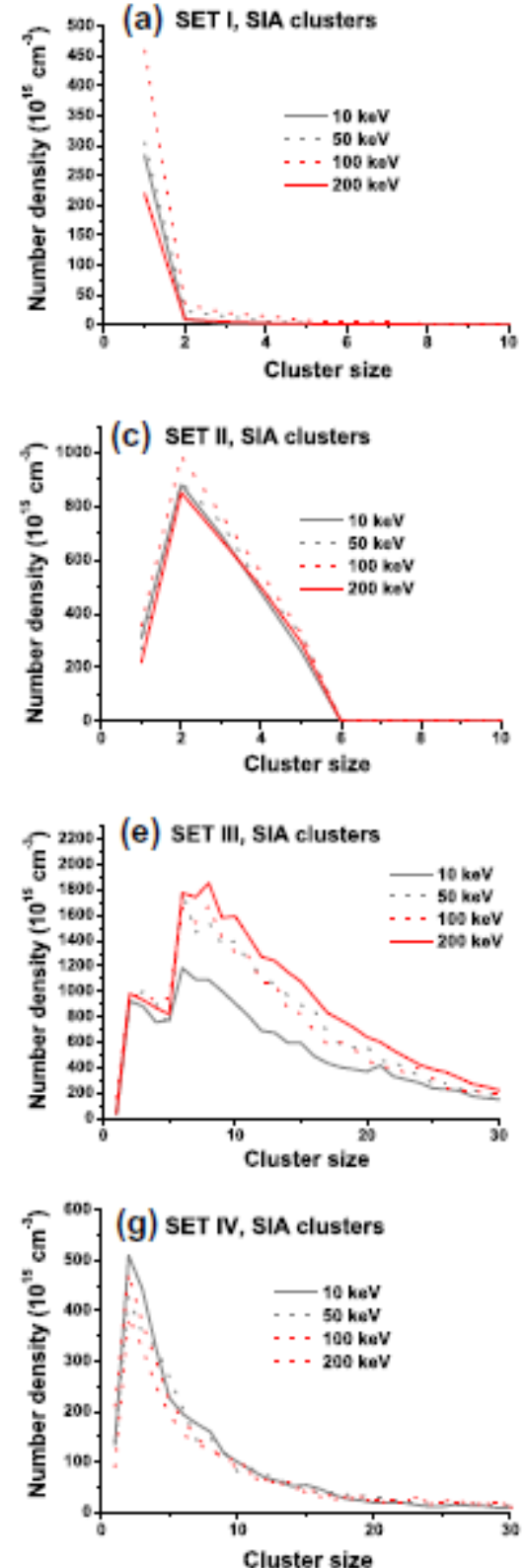

(b) SET I, VAC clusters
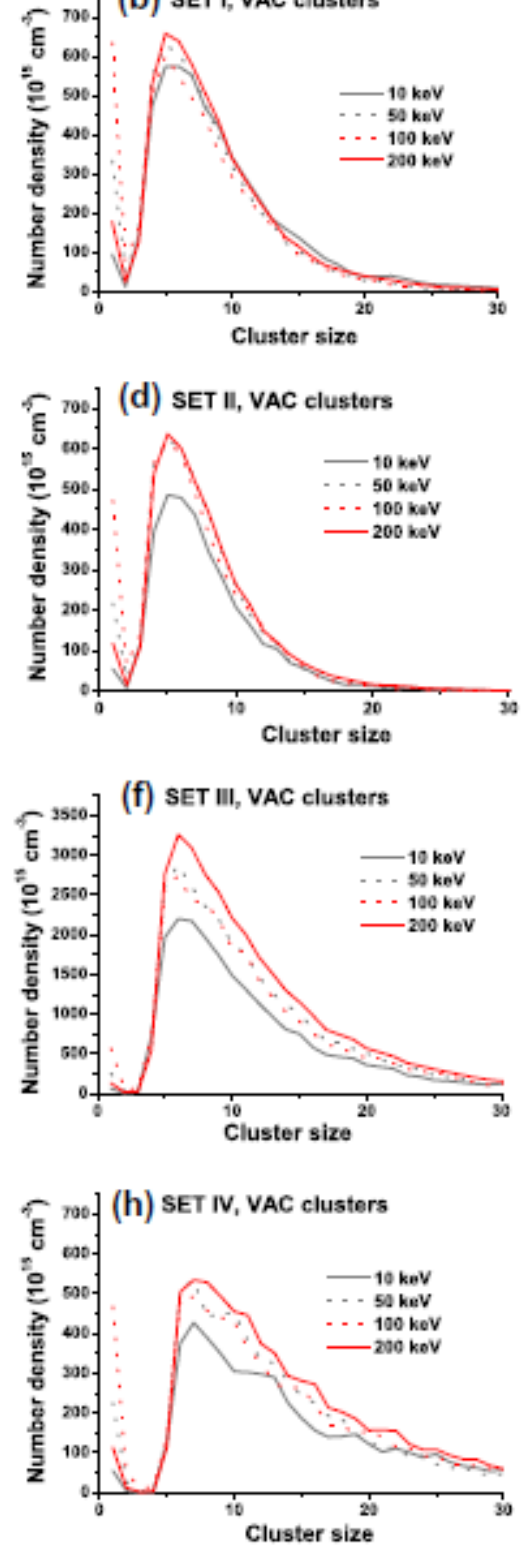

Figure 3: Cluster size distributions predicted at the end of 0.1 dpa irradiation as obtained with four sets of OKMC parameters using MD cascades with energies from $10 \mathrm{keV}$ to $200 \mathrm{keV}$ as input. The results obtained with cascades generated by 20,30 , and $40 \mathrm{keV}$, not represented, are undistinguishable from those obtained at $50 \mathrm{keV}$.From (Souidi et al. 2011) 


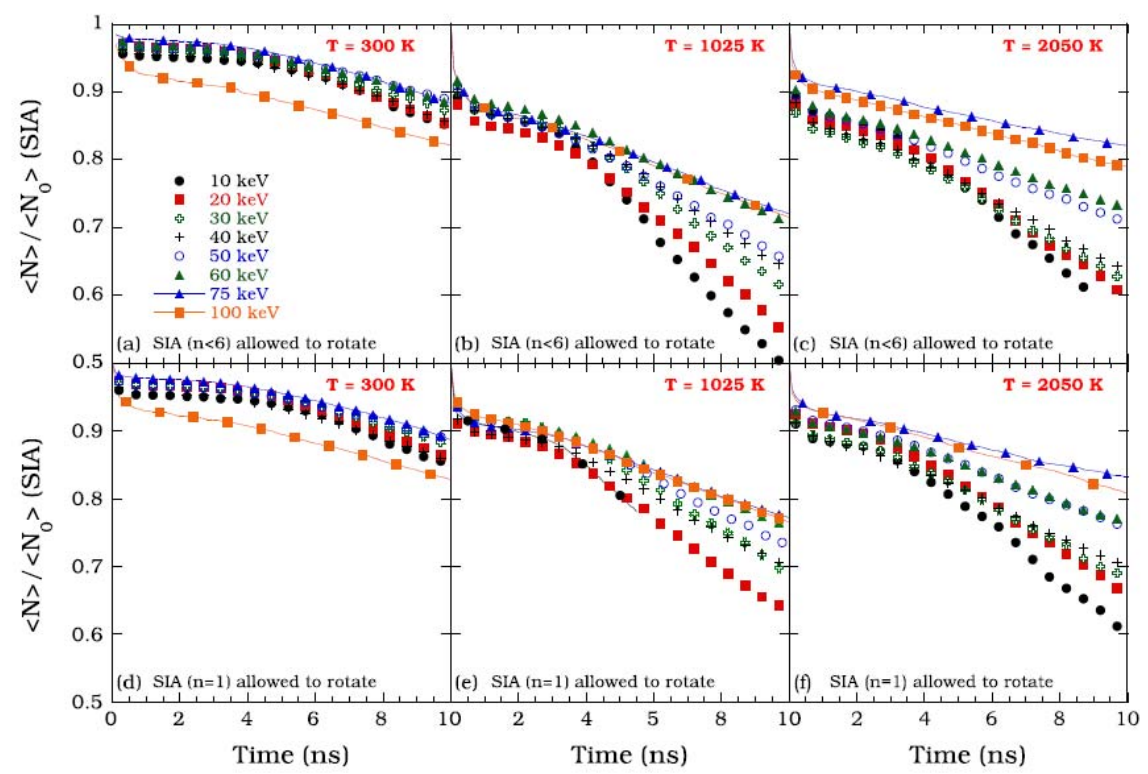

Figure 4: Surviving fractions of SIAs as a function of time for different PKA energies at 300, 1025 and $2050 \mathrm{~K}$, where all SIA clusters diffuse in 1D. In figure (a)-(c) only small SIA clusters $(\mathrm{n}<6)$ rotate. In figure (d)-(f) only mono-SIAs rotate. From (Nandipati et al. 2015b)

The main outcome of all these studies is to point out as in the previous section that it is necessary to have a better understanding of how the point defect and point defect clusters mobilities are affected by solutes, temperatures and other point defects.

\section{IV-2 Precipitation, segregation and phase transformations}

Point defect fluxes in the materials along with ballistic mixing can lead to phase transformations or segregations at extended defects. In the case of phases predicted by thermodynamics, the irradiation will just accelerate the phase transformation in what is referred to as irradiation enhanced phase transformation, a typical example is the formation of $\mathrm{Cu}$ precipitates in $\mathrm{Fe}$. In other cases, the irradiation induces the transformation into a non-equilibrium phase by changing for instance the solubility limit as has been observed in the $\mathrm{FeCr}$ system (Miller et al. 1996). kMC, and more precisely AKMC has been used to study phase transformations for more than twenty years first in binary ferritic alloys ( $\mathrm{FeCu}$ and $\mathrm{FeCr}$ ), then in more complex ones such as dilute Fe based alloys representative of RPV steels. Austenitic steels are much more complicated to model because they are paramagnetic and in metastable states. Deriving cohesive models of paramagnetic and / or metastable states is much more difficult, this probably explains why, at the moment, not that many KMC studies have been devoted to these alloys even though RIS is frequently observed at grain boundaries. For ferritic alloys, the historic case is the modelling of copper rich precipitates (CRPs) in bcc $\alpha-\mathrm{Fe} . \mathrm{Cu}$ is of primary importance in the embrittlement of the neutron-irradiated RPV steels. It has been observed to segregate into copper-rich precipitates within the ferrite matrix under irradiation. Since its role was discovered more than 50 years ago (Hornbogen and Glenn 1960), Cu precipitation in $\alpha$-Fe (Figure 5) has been observed by tomography, small angle neutron scattering, and high resolution transmission electron microscopy. Numerical simulation techniques such as rate theory or AKMC methods have also been used to investigate this problem (Soisson et al. 1996) (Wirth and Odette 1998) (Domain et al. 1999) (Schmauder and Binkele 2002) (Khrushcheva et al. 2003) (Djurabekova et al. 2007) (Monasterio et al. 2007) (Castin et al. 2009) (Pascuet et al. 2011) for a review see (Soisson et al. 2010). Other solutes present in RPV steels were more recently found to segregate and have been studied. In agreement with experimental 
observations, the addition of $\mathrm{Ni}$ has been found to promote the formation of a higher density of smaller CRPs in binary $\mathrm{Fe}-\mathrm{Cu}$ alloys, and copper-shell microstructures have been observed in $\mathrm{Fe}-\mathrm{Cu}, \mathrm{Mn}, \mathrm{Si}$ (Vincent et al. 2006; Bonny et al. 2009). Very recently it was found that Ni promotes the nucleation of $\mathrm{Cu}$ clusters and delays the precipitation kinetics during the coarsening stage because it slows down the $\mathrm{Cu}$ clusters (Wang et al. 2017) that are otherwise more mobile than isolated $\mathrm{Cu}$ atoms (Soisson and $\mathrm{Fu}$ 2007). More complex RPV based alloys have also been investigated (Liu et al. 1997) (Vincent et al. 2008a) (Ngayam-Happy et al. 2012) (Bonny et al. 2013), in particular to investigate the formation of the so-called late blooming phases (Odette and Nanstad 2009), phases that are supposed to start to form above a threshold incubation dose in low-Cu, high-Ni (and/or high-Mn) RPV steels, thereby causing additional hardening. 

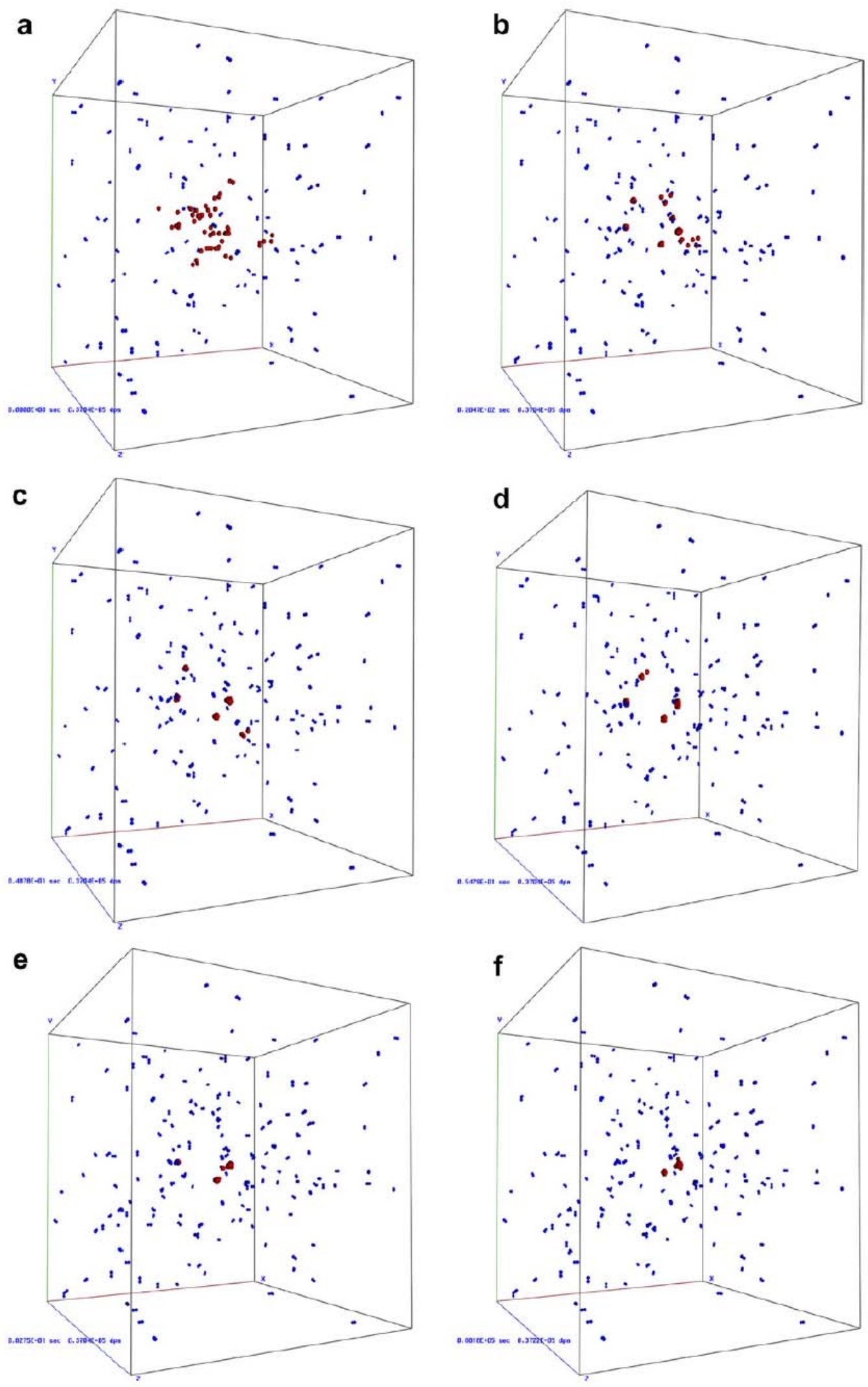

Figure 5 Representative vacancy (red circles) and clustered $\mathrm{Cu}$ atom (blue circles) evolution in an $\mathrm{Fe}-$ $0.3 \% \mathrm{Cu}$ alloy during the aging of a single $20 \mathrm{keV}$ displacement cascade, at (a) initial (200 ns), (b) 2 $\mathrm{ms}$, (c) $48 \mathrm{~ms}$, (d) $55 \mathrm{~ms}$, (e) $83 \mathrm{~ms}$, and (f) $24.5 \mathrm{~h}$. From (Monasterio et al. 2007). 
The second binary system most widely investigated is $\mathrm{FeCr}$, model material for high-Cr ferritic/martensitic steels. This system is quite complex as depending upon $\mathrm{Cr}$ concentration, precipitation or spinodal decomposition can take place and numerous AKMC simulations have been proposed (Wallenius et al. 2007) (Pareige et al. 2009) (Pareige et al. 2011) (Soisson and Jourdan 2016) (Soisson et al. 2018). Senninger et al. (Senninger et al. 2016) found that the diffusion of vacancies toward sinks leads to a Cr depletion, whereas the diffusion of self-interstitials causes an enrichment of $\mathrm{Cr}$ in the vicinity of sinks. The enrichment or depletion of $\mathrm{Cr}$ near sinks result from the balance of these two mechanisms and depend thus in a non simple manner on concentration and temperature. Avery recent study by Soisson et al. (Soisson et al. 2018) (Figures 6 and 7) pointed out the impact of the sink density on the delicate balance that exists between ballistic mixing and accelerated diffusion on the $\alpha$ ' transformation.

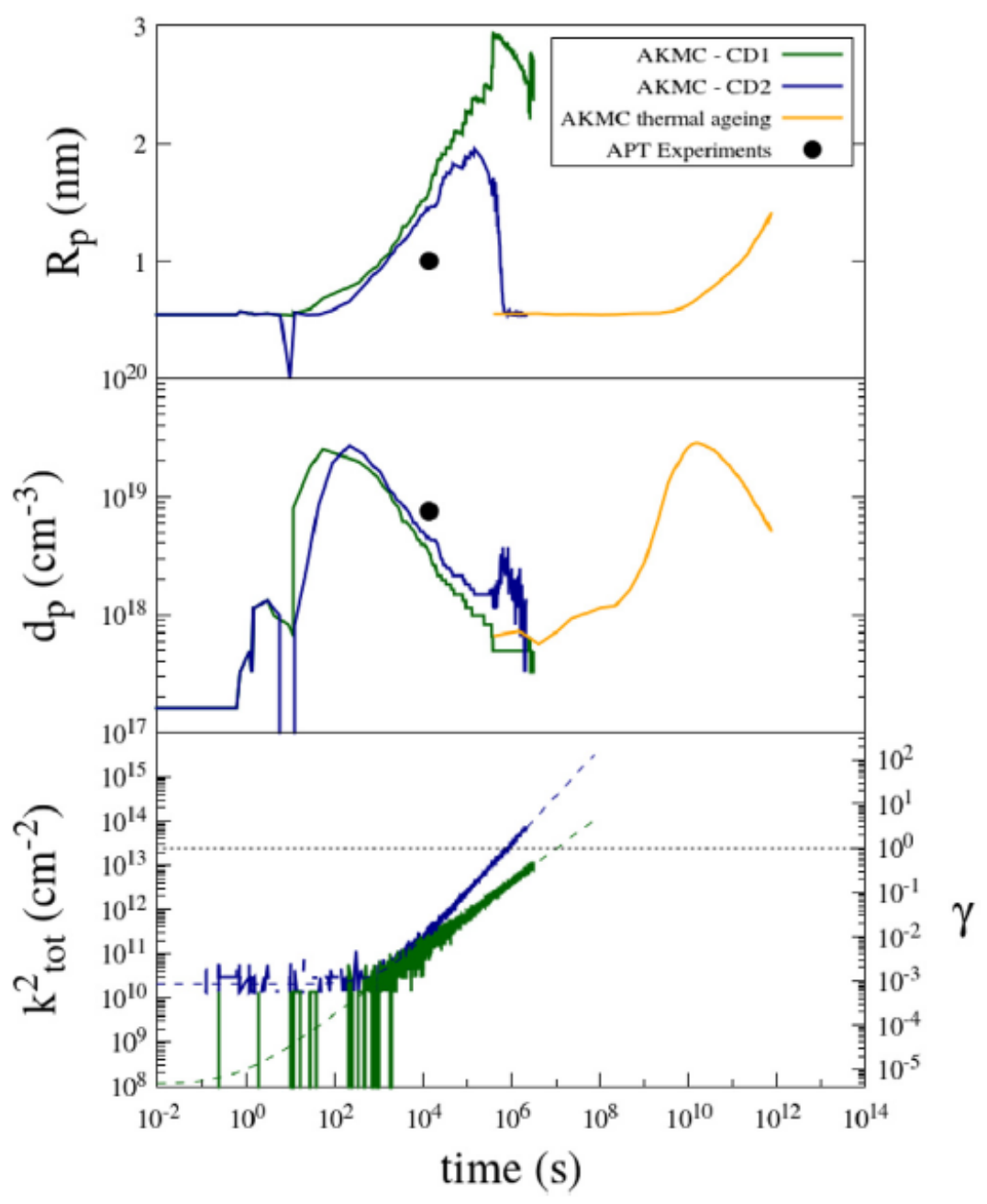

Figure 6: Evolution of the average radius and density of $\alpha^{\prime}$ precipitates, and of the total sink strength and irradiation intensity in a $\mathrm{Fe}-15 \% \mathrm{Cr}$ alloys under ion irradiation at $5.2 \times 10^{-5} \mathrm{dpa}$. $\mathrm{s}^{-1}$ and $300{ }^{\circ} \mathrm{C}$. Comparison between APT observations and AKMC simulations. CD1 and $\mathrm{CD} 2$ are two sets of parameters for the cluster dynamic method, used to compute the evolution of the sink density / sink strength. From (Soisson et al. 2018) 


$$
\mathrm{t}=1.69 \mathrm{e} 3 \mathrm{~s}
$$

\subsection{6e-004 dpa}

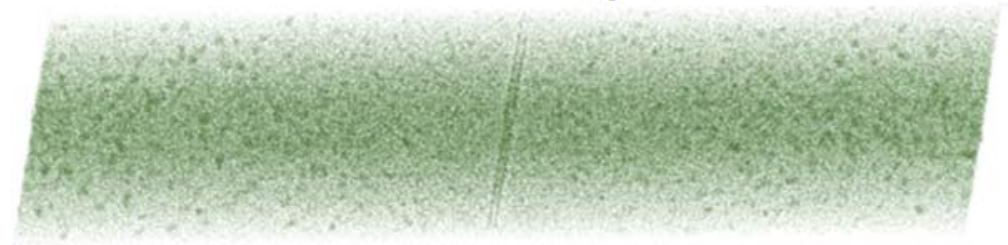

$\mathrm{t}=9.562 \mathrm{e} 3 \mathrm{~s}$

\subsection{5e-3 dpa}
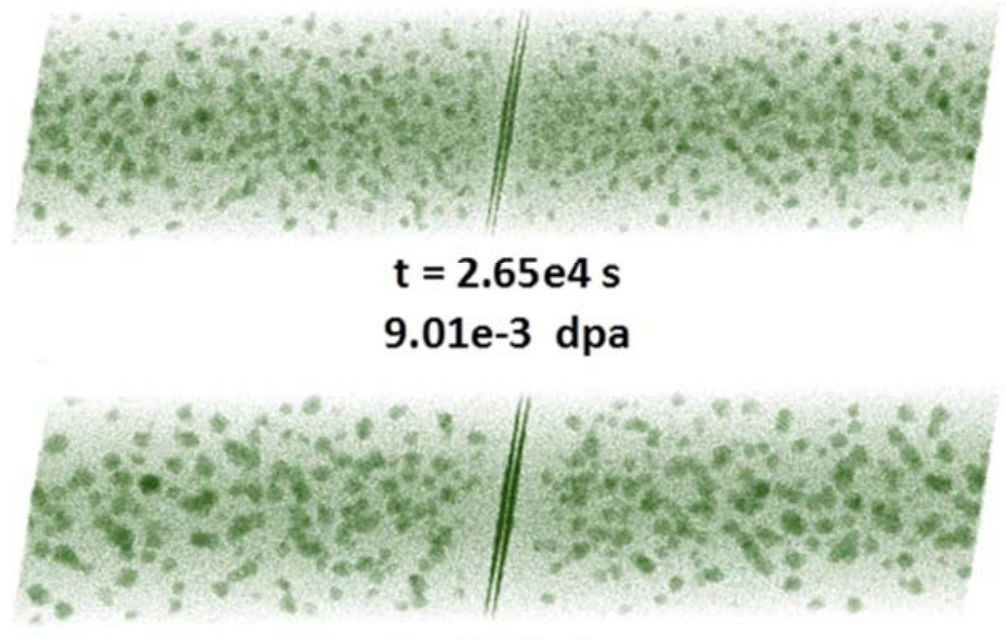

$$
\mathrm{t}=5.82 \mathrm{e} 4 \mathrm{~s}
$$

$1.98 \mathrm{e}-2$ dpa

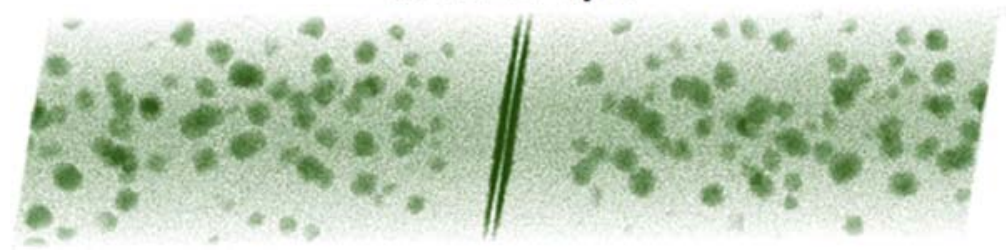

Figure 7: AKMC simulations of $\alpha-\alpha^{\prime}$ phase separation in $\mathrm{Fe}-15 \% \mathrm{Cr}$ under an irradiation at $290{ }^{\circ} \mathrm{C}$ and $3.4 \times 10^{-7} \mathrm{dpa}^{-1}{ }^{-1}$, on the grain boundary and on the neighboring planes. From (Soisson and Jourdan 2016)/

In $\mathrm{W}$, the formation of Re and Os through transmutation has inspired a few KMC (AKMC and OKMC) studies that show that Re and Os impact on the migration of the SIAs (Suzudo et al. 2015) (Huang et al. 2017). They control the nucleation and the growth of Re-rich clusters and also have a positive impact on swelling and void lattice formation (Bonny et al. 2018). Radiation induced segregation near extended defects (sinks) such as grain boundaries has also been investigated using KMC.

Other systems have been modeled to study phase transitions or precipitations. We can mention $\mathrm{ZrSiO}_{4}$ (Heinisch and Weber 2005), FeCrAl (Ejenstam et al. 2015), $\mathrm{Cu}_{83.5} \mathrm{Ag}_{15} \mathrm{~W}_{1.5}$. (Zhang et al. 2015), Cu-Nb$\mathrm{W}$ (Bellon and Averback 2015) Cu-W (Zhang et al. 2016). For a recent review of precipitation modelling by AKMC approaches see (Becquart and Soisson 2018). Note that a few studies have also been dedicated to investigate the stability of nanoparticles under irradiation and characterize their dissolution (Vu et al. 2015) (Vu et al. 2016). 
The fate of point defect fluxes towards sinks and their coupling with solutes is one of the most important issue of microstructure evolution under radiation and twenty years of AKMC studies have led to significant results such as the motion of $\mathrm{Cu}$ clusters (Soisson and $\mathrm{Fu} 2007$ ) and the synergy between solutes (Vincent et al. 2006; Bonny et al. 2009) (Wang et al. 2017). Synergy between solutes makes these studies very difficult as one must find good cohesive models for complex alloys which need not only to be accurate regarding the formation energies of the solute / point defect clusters but also for the migration energies of the point defects in these complex environments. Different approaches are currently being pursued (Becquart et al. 2018b) using for instance machine learning, but the task is not trivial, especially for multi-component alloys such as steels. For more simple alloys such as W-Re, or $\mathrm{Fe}-\mathrm{Cr}$, AKMC based on a large DFT data base, has really helped in unveiling mechanisms and explain experimental observations.

\section{IV-3 Typical damage accumulation/ microstructure evolution: impact of dose, temperature, impurities ...}

Many $\mathrm{KMC}$ studies has also been dedicated to characterize damage accumulation in various materials to model specific experiments such as ion implantation in Si for instance (Hobler and Otto 2003) or to investigate the impact of various parameters: the irradiation technique (ions versus neutrons (Castin et al. 2018), ions versus protons (Fluss et al. 2004), continuous versus pulsed (Perlado et al. 2003)), the neutron spectrum (Soneda et al. 2003) (Choi and Joo 2013) (Figure 8), the dose, and dose-rate (Soneda et al. 2003) (Chiapetto et al. 2016a), the temperature (Soneda et al. 2003) (Arévalo et al. 2007) (Chiapetto et al. 2016a) (Castin et al. 2018), impurities or solute atoms (Alonso et al. 2000) (Gámez et al. 2007) (Castin et al. 2018), the effect of annealing (Caturla et al. 2000b) (Fluss et al. 2004) or to compare materials (Caturla et al. 2000a). 

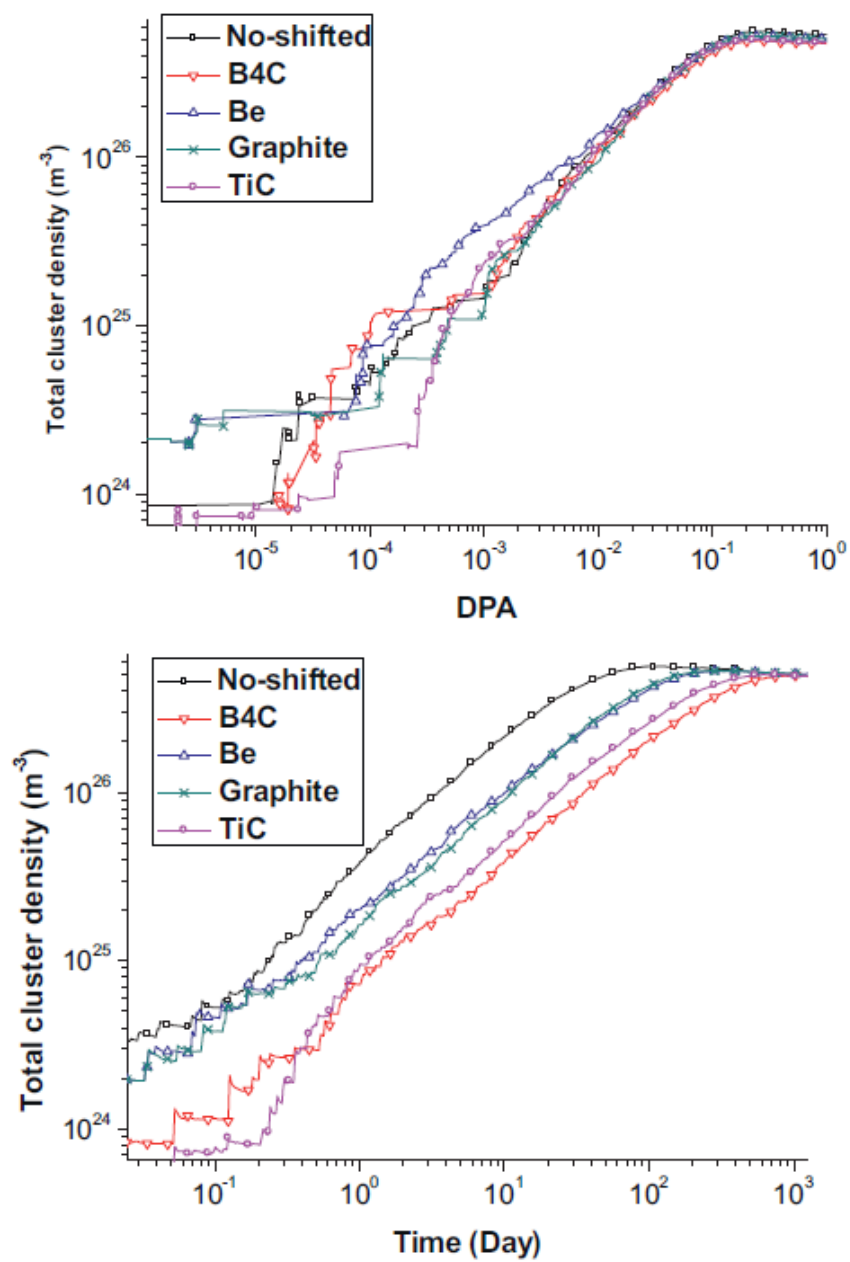

Figure 8: DPA and time evolution of total cluster density for different spectral shifters. From (Choi and Joo 2013)

The irradiation is typically modeled using cascade databases obtained either through MD (Perlado et al. 1998) (Caturla et al. 2000a) (Caturla et al. 2000b) (Wirth et al. 2001) (Marian et al. 2001) (Arévalo et al. 2007) (Chiapetto et al. 2016a) (Warrier et al. 2016) (Castin et al. 2018) or the BCA method (Hobler and Otto 2003) (Otto et al. 2005) (Hobler et al. 2005) (Becquart et al. 2006) (Valles et al. 2015) (Payet et al. 2016). The effect of pulsed irradiation has been studied in (Caturla et al. 2001) and showed that, in $\mathrm{Cu}$, the impact of the pulse depends on the frequency. Studying the effect of flux, (Diaz et al. 1997) showed that damage accumulation can be, in certain instances (room temperature and light ion implantation), reduced by decreasing the dose rate. The delicate balance between dose-rate and temperature has also been pointed out by (Soneda et al. 2003). This truly underlines the power as well as the weakness of KMC as the fate of the point defects injected by the irradiation cannot be simply deduced from the knowledge of their migration energies and many aspects must be considered. For instance, long term simulations of accumulation of cascades reveal the sensitivity of the system to the properties of the displacement cascades (their spatial extent, morphology and the spatial correlation of defects) in the evolution of point defect cluster size distributions and it was shown that the sensitivity can be low or strongly dependent on other parameters such as the mobility of defects, the type of interactions, the presence of impurities and the temperature (Becquart et al. 2006) (Souidi et al. 2011). A more recent study, comparing the damage accumulation in $\mathrm{W}$ obtained using BCA cascades or MD cascades finds that in the case of ion irradiation, BCA results can lead to good OKMC results despite 
the fact that BCA calculations do not take into account correctly the low energy events taking place in the cascades [chapter 00028]. However in the case of neutron irradiation, the study predicts that problems may arise when using the BCA cascades as this method overestimates the amount of FP created by high energy PKA (Valles et al. 2015) and it is thus mandatory to have proper ways of estimating the primary damage.

In the case of semiconductors, the materials are usually simpler, i.e. they do not contain as many alloying elements as structural materials such as steels. However, one has to take into account how electrons and holes distributions evolve along with the point defects. One possibility is to include them in an indirect manner as proposed in (Hehr 2014). In this approach, vacancies, interstitials, impurities, and reactionderived species (e.g. impurity/vacancy complexes) are treated as distinct species that are tracked individually as part of the KMC algorithm. Electrons and holes are assumed to exist in certain background concentrations (which depend on position in the device, operating characteristics, etc.), and their interaction rates with defects are quantified through analytic expressions that are functions of the carrier concentrations. These interaction rates are then included in the KMC event selection process, so that defect migration and carrier interaction are both taking place in the simulation. Electrons and holes are thus included in the simulation, but they are not tracked as individual particles that are subject to stochastic behavior. The method was used to model annealing of electron Irradiation of p-Type Silicon and good agreement between the model and the experiment was obtained.

\section{IV-4 Solute or impurity / gas atoms diffusion and the formation of gas bubbles}

Another topic which has been investigated for some time using $\mathrm{KMC}$ approaches is the modeling of $\mathrm{H}$ and $\mathrm{He}$ atoms in structural materials such as $\mathrm{Fe}$ and $\mathrm{W}$. This topic is quite important for fusion as structural materials that will be close to the plasma will be bombarded by $\mathrm{He}$ and $\mathrm{H}$ isotopes. In the case of $\mathrm{H}$ and $\mathrm{H}$ isotopes, the major concern is to obtain effective diffusion coefficients in the presence of extended defects (Ramasubramaniam et al. 2008) (Ito et al. 2017), hydrogen concentration (Yang and Oyeniyi 2017) or impurities (Oda and Tanaka 2011) in $\mathrm{Li}_{2} \mathrm{O}$. Diffusion of $\mathrm{H}$ has also been investigated on a W (001) reconstructed surface (Yang and Hassanein 2014). In the case of helium, the goal is often to model the formation of helium vacancy clusters that will be nuclei for voids and bubbles leading to swelling, formation of pores, blistering or exfoliation of the surface and embrittlement. He is a very mobile element in metals and forms very stable clusters which are also very mobile unless they are trapped by vacancies or other elements of the microstructure, dislocations, grain boundaries, solute atoms ... [chapter 00006]. Predicting the evolution of microstructures implanted by $\mathrm{He}$ atoms or containing $\mathrm{He}$ atoms is not straightforward as it depends on temperature, point defect and helium implantation rates, doses (Martínez et al. 2015) as well as the way they are implanted (at the same time, or in successive events, continuously or pulsed) or where they are implanted (close or not to free surfaces, i.e. in stress free material or in stress gradients). KMC simulations have investigated the fate of He during co-implantation in Fe and showed that at most $3 \%$ end up in vacancies (thus becoming substitutional) in typical implantation set ups (Erhart and Marian 2011). Another study demonstrated the occurrence of different regimes of He clusters nucleation in $\mathrm{W}$, one dominated by He self-trapping (at low temperatures and high implantation rates), the other one, at high temperatures and low implantation rates, where the trapping of helium by vacancies is predominant (Yang et al. 2017). Stress gradients due to the presence of surfaces were found to drive collective bubble populations towards the free surface or up the stress gradient (Sharafat et al. 2009) (Figure 9). Comparing continuous $\mathrm{He}$ implantation and pulsed $\mathrm{He}$ implantation, Rivera et al. found that the pulsed modes lead to a higher $\mathrm{He}$ retention at high temperature because they produce a higher concentration of defects (Rivera et al. 2013). 
Other simulations underline the role of point defects, and in particular the vacancies, and impurities (C for instance) that can trap the He atoms and thus impact on the size distribution of He clusters or on the rate of He desorption (Morishita et al. 2003) (Caturla et al. 2003) (Bringa et al. 2003) (Wirth and Bringa 2004) (Deo et al. 2007) (Caturla et al. 2008) (Becquart and Domain 2009) (Guo et al. 2013) (De Backer et al. 2015) (Perez et al. 2017). Based on the same ideas that He interacts quite strongly with point defects, other studies focus more on the impact of $\mathrm{He}$ atoms on the long term evolution of the primary damage and the formation of voids (Bringa et al. 2003) (Galloway and Ackland 2013) (De et al. 2013). All these studies underline the complex balance between point defect and helium clustering, cluster growth, trapping and detrapping reactions that lead to different microstructure evolutions. Most of these investigations have been done in bcc Fe and W, but a few studies in fcc metals (Figure 10) and even austenitic steels are available (Caturla et al. 2003) (Gámez et al. 2009) (De Backer et al. 2015). A preliminary study comparing the formation of mixed He vacancy clusters in bcc and fcc Fe seems even to find no major difference in clustering behavior (Suzudo et al. 2010). Finally KMC has been used to elucidate the formation of fuzz in $\mathrm{W}$, this peculiar nanostructured morphology, that was shown to result again from the balance between clusters formation and detrapping reactions (Suzudo et al. 2010)(Suzudo et al. 2010)(Suzudo et al. 2010)(Valles et al. 2017a). 

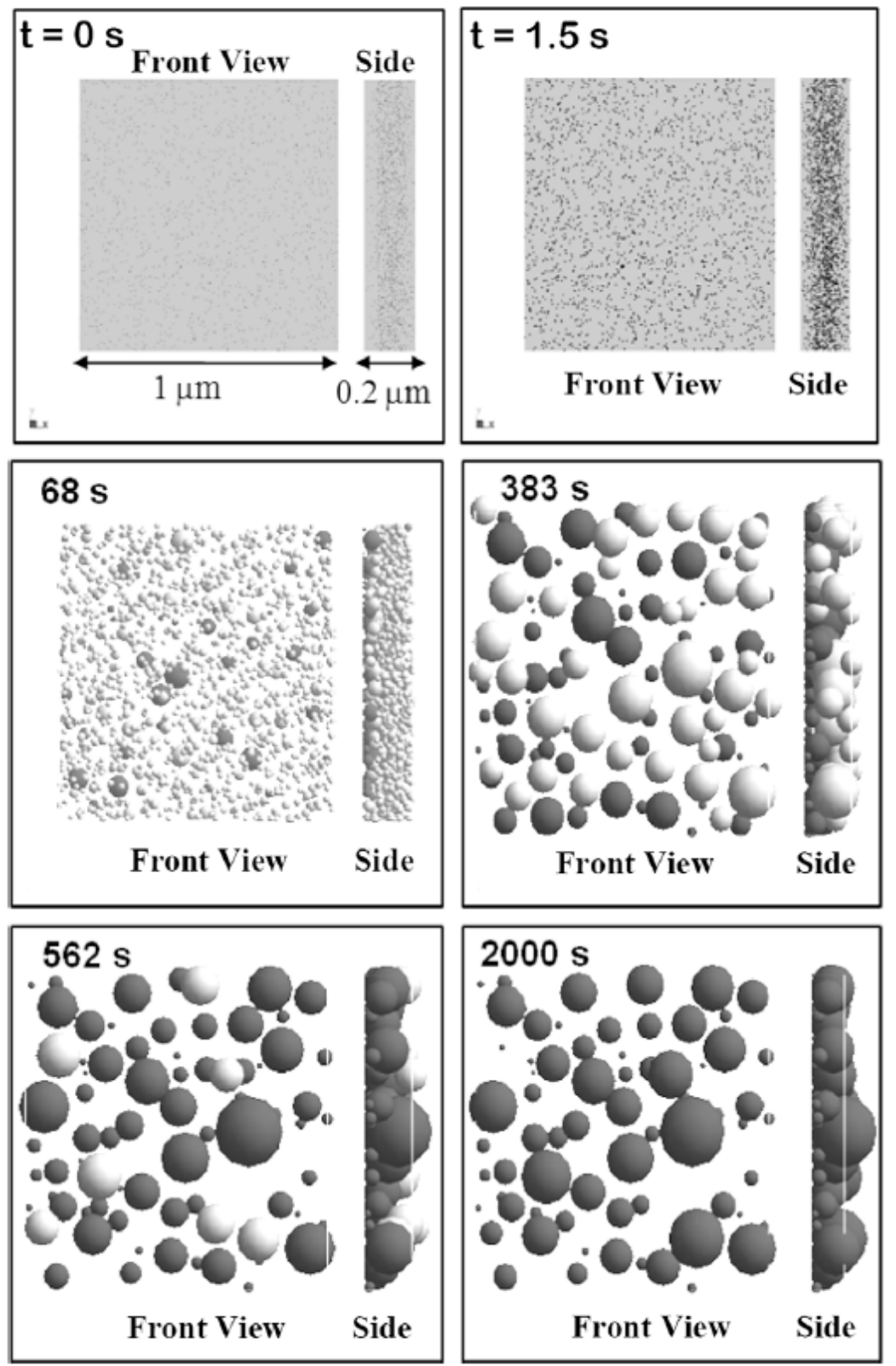

Figure 9: Snap shots of the time sequence of the KMC simulation results of the IEC facility at UWMadison helium implantation condition at $\mathrm{T}=730^{\circ} \mathrm{C}, 1.6 \times 10^{18} \mathrm{He} / \mathrm{m}^{2} \mathrm{~s}, \mathrm{E}_{\mathrm{He}}=30 \mathrm{keV}$; (light colored spheres represent matrix or bulk bubbles; dark spheres represent surface pores or bubbles that have penetrated the top surface). From (Sharafat et al. 2009) 


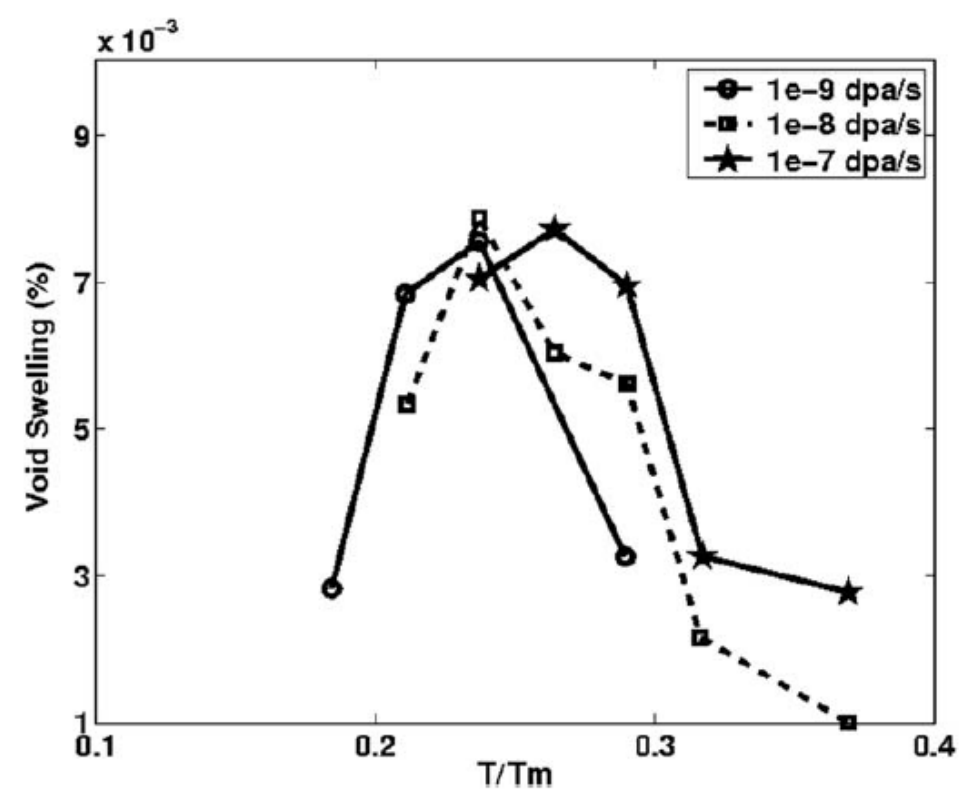

Figure 10: Void swelling (or change in volume due to vacancies in $\mathrm{He}-\mathrm{V}$ complexes) as a function of temperature for a total dose of $0.03 \mathrm{dpa}$ and three different dose rates for damage produced in $\mathrm{Au}$. From (Caturla et al. 2006)

In fuel materials, and particularly in $\mathrm{UO}_{2}$ it is the diffusion of oxygen and other gases which is mostly being investigated (Oaks et al. 2011) (Behera et al. 2016) (Maillard et al. 2016). See (Bertolus et al. 2015) for a review paper on the subject. Fission products, such as Ag in TRISO fuels (Méric de Bellefon and Wirth 2011) has also been studied, though not really extensively it seems.

The formation and diffusion of gas bubbles has a large impact on structural as well as fuel materials and despite an already quite large amount of studies dedicated to this, more work is needed because of the delicate balance between point defect and gas atom clustering properties that depend, of course, on the matrix and all its solutes or alloying elements but also on internal pressure, to which they will contribute. In that regard, elastic interactions must be accounted for in the models when bubbles start to grow which adds another level of complexity to the models.

\section{IV-5 Investigating solutions to eliminate the point defects or trap gas atoms.}

Radiation damage in metallic alloys consists, as already mentioned, mostly in a large amount of point defects that will diffuse, leading to many changes such as RIS, the formation of solute clusters, the formation of voids and dislocation loops etc. All these features decrease the mechanical properties of the components and solutions are thought to trap or eliminate the point defects. In that search, KMC has been used to evaluate the impact of grain boundaries on the fate of point defects in $\mathrm{Ni}$ and a $\mathrm{Ni}$-Cr alloy showing that both vacancies and SIAs are attracted to the grain boundaries (Al Tooq and Kenny 2013). A later study, showed that, in Fe, both near GB and at GB, low-energy-barrier/barrier-free regions form around the interstitials, promoting the annihilation of vacancies (Li et al. 2016). Comparing single crystal tungsten and mono crystal tungsten, Valles et al (Valles et al. 2017b) confirm the impact of GB on the on the amount and distribution of vacancies as well as on the migration and retention of $\mathrm{H}$ and propose that GB act as preferential path for $\mathrm{H}$. Another study, in Al, showed, on the other hand, that diffusion of $\mathrm{H}$ was decreased by more than one order of magnitude in GBs (Pedersen and Jónsson 2009). In $\mathrm{UO}_{2}$, it was found that certain types of grain boundaries enhanced the mobility of $U$ vacancies (Uberuaga and Andersson 2015) (Figure 11). An increase of the diffusivity of Ag and Cs in Tristructural-Isotropic (TRISO) fuel particles in high energy GB as compared to the bulk was also found by Ko et al. (Ko et al. 2016) (Ko et al. 2017). The apparent contradictions in these results stem from the fact that depending 
on several factors such as the grain size, the GB energy, angle, the probability of GB connectivity, and the excess of free volume, GB can be diffusion enhancer or diffusion slower as shown recently in the case of $\mathrm{H}$ diffusion in $\mathrm{Ni}$ (Oudriss et al. 2012). Martensite laths was investigated using OKMC simulations and was found not to be the reason for the different nanostructural evolution under neutron irradiation of ferrite and martensite in $\mathrm{FeCrC}$ alloys (Chiapetto et al. 2016b).
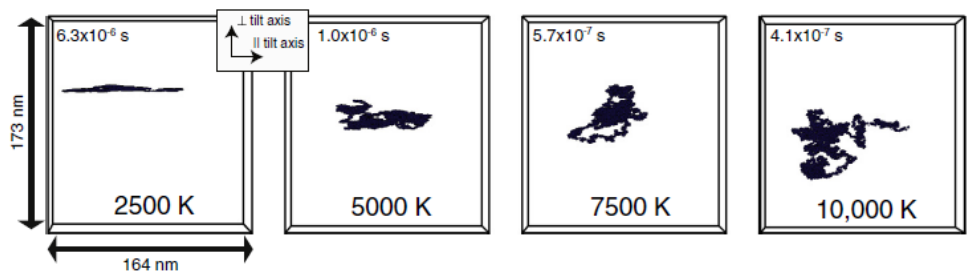

Figure 11: Example trajectories at each temperature considered $(2500 \mathrm{~K}, 5000 \mathrm{~K}, 7500 \mathrm{~K}$, and 10,000 $\mathrm{K})$ for a vacancy diffusing at the R5 tilt GB. There is a clear transition from one-dimensional to twodimensional motion as the temperature is increased. The direction along the tilt axis is indicated for clarity. The time scale for each simulation is also indicated in the upper left corner of each frame. From (Uberuaga and Andersson 2015).

Directly related to the issue of trapping / eliminating point defects or gas atoms in extended defects is the determination of the sink strength, a parameter typically used in the Mean Field Rate Theory (MFRT) [chapter 00022] to describe the interaction of migrating defects with the features characterizing the microstructure of the material (e.g. voids, dislocations, grain boundaries). Because KMC approaches explicitly take into account spatial correlations between the elements of the physical system, they are expected to implicitly reproduce the effect of sinks or traps for migrating species. They have thus been used for more than twenty years to determine the sink strength of various extended defects and compare them to analytical approaches (Heinisch et al. 2007) (Malerba et al. 2007) (Jansson et al. 2013) (Hou et al. 2016) (Ahlgren and Bukonte 2017) (Carpentier et al. 2017) (Sivak et al. 2015) (Vattré et al. 2016) (Martínez et al. 2018).

The results summarized above highlight one strength of KMC methods: its capacity to investigate mechanisms at the atomistic level in microstructures containing extended defects on time scales longer than MD. Despite the fact that the results depend on the point defect and point defect clusters mobility, and / or on the cohesive model used, they show where more precise studies should concentrate on. A typical example is the impact of grain boundaries which is more complicated than initially thought as grain boundaries can be seen as containing a large population of defects with rich and largely not understand behavior and impact. Off lattice on-the-fly AKMC should be able to provide valuable information, for systems for which a good cohesive model and a lot of computing time are available. They could help build databases of grain boundary families useful to be integrated in mean-field approaches.

Another family of extended defects which has been barely considered are surfaces probably because of the difficulty to model, even at the atomistic scale, the complexity of real surfaces (their roughness, the oxide layers, the strain gradients...). Furthermore, reactions occurring at surfaces take place at quite different time scales, which is a problem for KMC techniques similar to the low barrier problem mentioned in section II-2 On-the fly kMCs. For some problems, such as the formation of solute clusters under irradiation, surfaces may not be important, for others such as the formation and diffusion of gas bubbles, it will be difficult not to investigate this issue. 


\section{Comparison of models}

The formation and growth of point defect and solute clusters through all stages of cluster evolution, from their nucleation to growth and coarsening has been extensively simulated by two alternate methods, mean field reaction rate theory (MFRT) [chapter 00022] and kinetic Monte Carlo at the mesoscale level, i.e. OKMC. In principle, these two methods should predict essentially the same behavior when used to solve the same problem, if the problem is well posed as shown in (Stoller et al. 2008) but that the comparison is not straightforward and needs to be done very carefully. One important conclusion of the work by Stoller et al. (Stoller et al. 2008) is that, because of computational limitations, for the time being, one immediate application of OKMC simulations is to improve the parameterization of the MFRT models as shown in section IV-2. A similar conclusion was drawn by (Rottler et al. 2007) who compared AKMC and MFRT in the modeling of RIS. This situation may evolve as a recent development allowing to speed up KMC simulations enabled Xu et al. (Xu et al. 2012a) to model the entire radiation process of molybdenum nanofoils irradiated in a transmission electron microscope.

\section{Coupling with other methods}

To model radiation damage, the different codes necessary to go from the neutron spectra to the evolution of the mechanical or thermal properties of the material (Becquart and Domain 2011) are typically used independently. In that scheme, one code provides the input data as shown in section IV-3 or in (Sharafat et al. 2009) or parameters (DFT and / or MD results concerning the stability and migration of point defects and point defect clusters in KMC models for instance) for the next, in a coarse graining multiscale approach (Jumel et al. 2002). However, a few attempts at using different approaches in the same model have been developed. For instance, the OKMC approach is sometimes used within a lower scale method as proposed in (Castin et al. 2011) and later (Pannier 2017) where the AKMC is coupled with the OKMC in the same code, thus producing an hybrid AKMC-OKMC code (Domain and Becquart 2019).

\section{Locks / things that need to be investigated / improved further}

In $\mathrm{MD}$, the determining parameter is the quality cohesive model that controls the validity of the thermodynamic and dynamic properties (Becquart et al. 2000). As a consequence, MD simulations are far from being capable of representing real microstructures that always contain impurities that are not described properly by current force fields. With the help of KMC models, many studies have demonstrated that the mobility of point defects, the stability of the various objects that can form (point defect clusters, mixed He point defect clusters, dislocations and dislocations loops etc) as well as their mobility, the trapping or slowing down capability of impurities as well as the impact of grain boundaries have tremendous effects. These models are thus capable of providing relevant information that closer to real materials. However, these simulations require that a large number of parameters (and the way they evolve with temperature) be known accurately, not to mention the impact of surfaces (Aliaga et al. 2016) (Duan et al. 2018) or the source term, as recently shown in (Jourdan and Crocombette 2018). This means that considerable effort must go into choosing the level of details to enter in the models to minimally perturb the results. For instance a recent study showed that the additional complexity of size dependent vacancy emission rates from voids had little effect on the defect concentrations (Hoffman et al. 2016). The authors conclude thus that a constant barrier should be sufficient for simulations of voids in bcc metals. 
Nevertheless, even if KMC allows to model the nucleation and growth of larger defects than MD, comparing with experimental data is still a challenge and it is necessary to build tools that link the experimental observations to the objects formed in the simulations. A typical example is the electrical resistivity (ER) method which can provide valuable information on the migration, clustering and dissociation of radiation defects, which are essential for the behavior of materials under irradiation. The ER method has been widely employed due to its high sensitivity, reliability and experimental convenience. Despite the importance of the ER method for radiation damage studies, the quantitative analysis of experimental results is still hindered by the lack of detailed knowledge on the specific contributions from different types of radiation defects, e.g., point-defects, point-defect clusters or defectimpurity complexes to the total resistivity. So far, the crude assumption is made that each point defect (isolated or in cluster) has the same contribution to the electrical resistivity. This is clearly a strong approximation and a better estimate of point defect and point defect cluster residual resistivity will be very helpful to compare the results of KMC and RE models with experiments.

If better experimental data is needed at the atomic level, a number of issues limit the applications of AKMC. First, the description of the basic kinetic steps, be it from ab initio or empirical description or fully phenomenological. At the moment, no approach provides a general answer, either because costs are too large or the information is not available. There a number of efforts to improve the various levels of description, many involving machine-learning, but the problem remains largely open today. A second and related issue focuses on computing rates accurately, a challenge even with accurate force fields. Given the cost of this step, a number of different approximations have been proposed. Recent simulations suggest that these rates could vary even more than previously thought, which forces a reexamination of many of those used until now. More technically, the reachable simulation time is an important third challenge: many phenomena take place on time scales that are beyond current methods, even if one is ready to wait for days or weeks. To overcome this limitation, efforts have gone, for example, into parallelizing time evolution for large KMC simulations (Martínez et al. 2008) (MartinBragado et al. 2015) (Wu et al. 2015) (Jiménez and Ortiz 2016). These approaches are technically complex and are limited to relatively system problems. Other approaches have focused on managing flickers, i.e., the low-energy barrier mechanisms, to allow rare events to take place. Different methods are being developed to boost the simulation by solving analytically part of the time evolution, see for instance (Donev et al. 2010), (Athènes and Bulatov 2014) or (Danielson et al. 2017). A final challenge is linked to the capacity at describing a system in its full complexity, or, at least, with all the complexity needed to address a specific challenge. Although progress has been made in understanding the role of impurities, defects, surfaces, strain, etc., introducing structures at many different length scales remains challenging.

Another issue that slows done the simulations is the calculation of the transition rates. Machine learning can speed up these calculations as proposed in (Domingos et al. 2006) (Djurabekova et al. 2010) (Castin and Malerba 2010), as well as be used to help building better cohesive models [chapter 00019] (Brommer et al. 2015) (Behler 2016) (Messina et al. 2017).

\section{Conclusions}

$\mathrm{KMC}$ approaches are techniques useful to study generic as well as realistic problems. It provides the link between MD and MFRT (Figure 12); the AKMC being close to the former, whereas OKMC and EKMC are close to the later. The same algorithm (time residence) can provide different information depending upon the method chosen and the approximations. A typical example is the impact of solutes 
and alloying elements. In the AKMC approach, solutes are introduced explicitly (a recent review of the different ways of doing so can be found in (Becquart et al. 2018b)) and the impact of radiation on the solute distribution can be estimated, and the structure and content of the phases or clusters formed can be obtained. If one choses the OKMC or EKMC with a grey alloy approach, the structure of the clusters is an entry of the model and not a result and it will not be possible to study phase transitions, whereas it will be possible to study the behavior of point defect fluxes in these alloys on longer time scales than when using AKMC.

The technique has been applied to investigate many issues in the radiation damage community, the most important ones being radiation induced or accelerated phase transformations, the annealing as well as the accumulation of the primary damage with or without gas atom diffusions and the search for solutions to remedy to the damage.

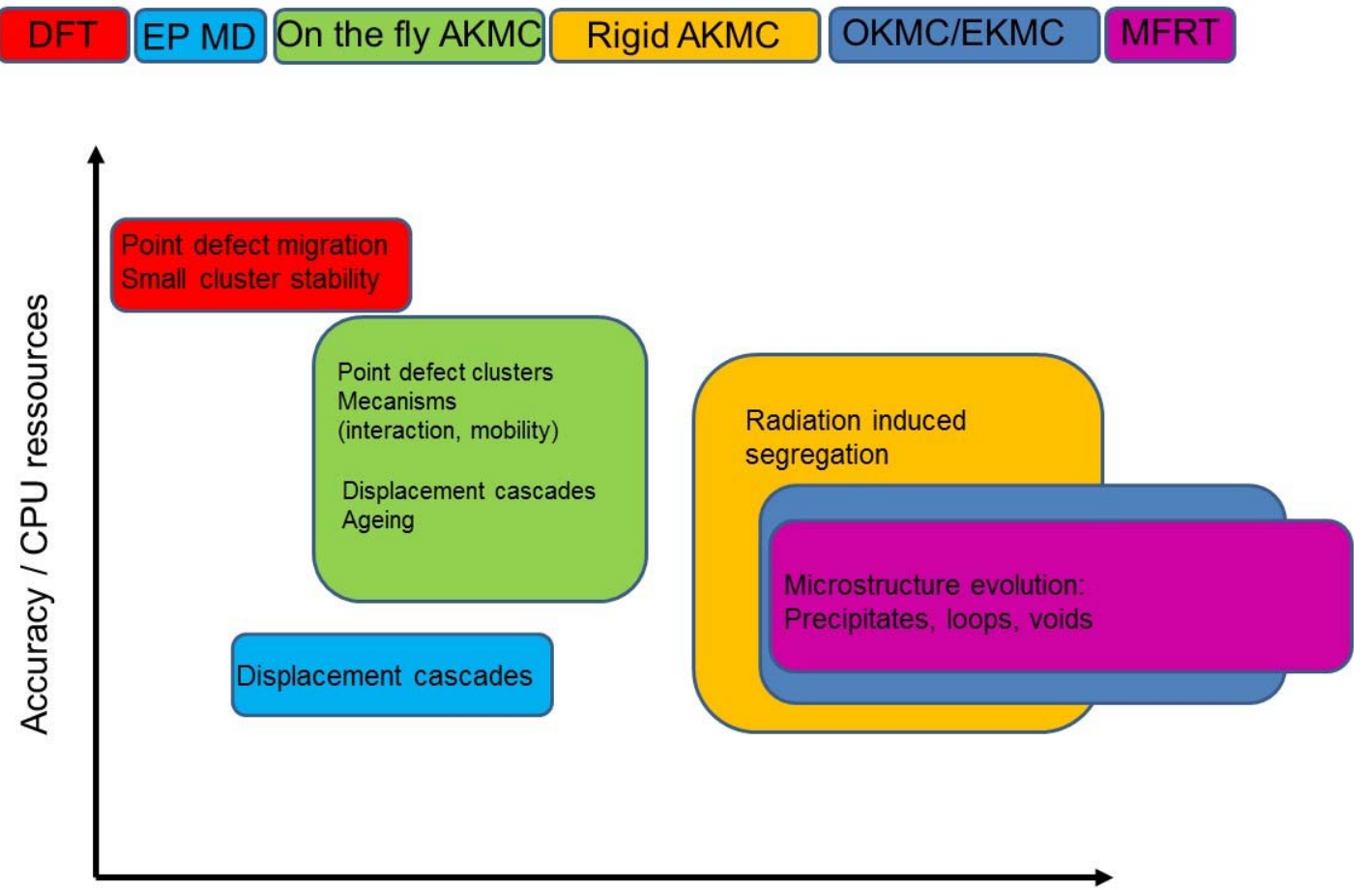

Lengthscale / Timescale

Figure 12: summary of the different KMC approaches compared to DFT and MD with empirical potentials.

The development of KMC approaches is continuing strongly at the moment at all approximation levels, with advances in the calculation of prefactors, the coupling of various approximations and attempts to use machine learning to facilitate event catalog building, strengthening the capacity of KMC methods to address a wider range of fundamentally and technologically relevant materials questions.

Yet, one of the main outcome of this chapter is to underline that using KMC techniques to their best abilities requires that one finds a good compromise between methods (lattice / of lattice; atomic / object) and precision required (for the cohesive model / parameterization) and calculation time. This is indeed similar to many modelling techniques, however because KMC is more flexible and offers a richer set of approximations and tools to choose from than, let's say, MD and MFRT, the expertise of the researcher is more crucial. This efforts, as we have shown in this chapter, is well worth it: well used, KMC techniques can clearly provide unique and essential understandings that cannot be achieved with other simulation methods at the moment. 


\section{References}

Ahlgren T, Bukonte L (2017) Sink strength simulations using the Monte Carlo method: Applied to spherical traps. J Nucl Mater 496:66-76. doi: 10.1016/j.jnucmat.2017.09.006

Al Tooq Z, Kenny SD (2013) Modelling radiation damage at grain boundaries in fcc Nickel and Nibased alloy using Long Time Scale Dynamics Techniques. Nucl Instrum Methods Phys Res Sect B Beam Interact Mater At 303:9-13. doi: 10.1016/j.nimb.2012.10.027

Aliaga MJ, Dopico I, Martin-Bragado I, Caturla MJ (2016) Influence of free surfaces on microstructure evolution of radiation damage in $\mathrm{Fe}$ from molecular dynamics and object kinetic Monte Carlo calculations: Influence of free surfaces on microstructure evolution of radiation damage in $\mathrm{Fe}$. Phys Status Solidi A 213:2969-2973. doi: 10.1002/pssa.201600158

Allen MP, Tildesley DJ (2017) Computer simulation of liquids: Second edition

Alonso E, Caturla M-J, Díaz de la Rubia T, Perlado JM (2000) Simulation of damage production and accumulation in vanadium. J Nucl Mater 276:221-229. doi: 10.1016/S0022-3115(99)00181-6

Arakawa K, Ono K, Isshiki M, et al (2007) Observation of the One-Dimensional Diffusion of Nanometer-Sized Dislocation Loops. Science 318:956-959. doi: 10.1126/science.1145386

Arévalo C, Caturla MJ, Perlado JM (2007) Temperature dependence of damage accumulation in $\alpha$ zirconium. J Nucl Mater 367-370:338-343. doi: 10.1016/j.jnucmat.2007.03.131

ASTM E693 (1994) E 693 Annual Book of ASTM standards. In: Annual Book of ASTM standards, ASTM. ASTM, Philadelphia, PA

Bacon DJ, Osetsky YN, Rodney D (2009) Chapter 88 Dislocation-Obstacle Interactions at the Atomic Level. In: Dislocations in Solids. Elsevier, pp 1-90

Barbu A, Becquart CS, Bocquet JL, et al (2005) Comparison between three complementary approaches to simulate ' large' fluence irradiation: application to electron irradiation of thin foils. Philos Mag 85:541-547. doi: 10.1080/14786430412331334616

Barkema GT, Mousseau N (1996) Event-Based Relaxation of Continuous Disordered Systems. Phys Rev Lett 77:4358-4361. doi: 10.1103/PhysRevLett.77.4358

Becquart CS, De Backer A, Domain C (2018a) Atomistic modelling of radiation damage in metallic alloys, Springer. Hsueh C.H., Schmauder S., Chen C.S., Chawla K.K., Chen W. ,Kagawa Y., Singapore

Becquart CS, Domain C (2010) Introducing chemistry in atomistic kinetic Monte Carlo simulations of Fe alloys under irradiation. Phys Status Solidi B 247:9-22. doi: 10.1002/pssb.200945251

Becquart CS, Domain C (2009) An object Kinetic Monte Carlo Simulation of the dynamics of helium and point defects in tungsten. J Nucl Mater 385:223-227. doi: 10.1016/j.jnucmat.2008.11.027

Becquart CS, Domain C (2011) Modeling microstructure and irradiation effects. Metall Mater Trans Phys Metall Mater Sci 42:852-870. doi: 10.1007/s11661-010-0460-7

Becquart CS, Domain C, Legris A, Van Duysen JC (2000) Influence of the interatomic potentials on molecular dynamics simulations of displacement cascades. J Nucl Mater 280:73-85. doi: $10.1016 / \mathrm{S} 0022-3115(00) 00029-5$ 
Becquart CS, Domain C, Malerba L, Hou M (2005) The influence of the internal displacement cascades structure on the growth of point defect clusters in radiation environment. Nucl Instrum Methods Phys Res Sect B Beam Interact Mater At 228:181-186. doi: 10.1016/j.nimb.2004.10.031

Becquart CS, Mousseau N, Domain C (2018b) Atomistic Kinetic Monte Carlo and solute effects, (C) Springer International Publishing AG, part of Springer Nature. W. Andreoni, S. Yip (eds.), Singapore

Becquart CS, Soisson F (2018) Monte Carlo Simulations of Precipitation Under Irradiation. In: Schmauder S, Chen C-S, Chawla KK, et al. (eds) Handbook of Mechanics of Materials. Springer Singapore, Singapore, pp 1-29

Becquart CS, Souidi A, Domain C, et al (2006) Effect of displacement cascade structure and defect mobility on the growth of point defect clusters under irradiation. J Nucl Mater 351:39-46. doi: 10.1016/j.jnucmat.2006.02.022

Behera RK, Watanabe T, Andersson DA, et al (2016) Diffusion of oxygen interstitials in UO2+x using kinetic Monte Carlo simulations: Role of $\mathrm{O} / \mathrm{M}$ ratio and sensitivity analysis. J Nucl Mater 472:89-98. doi: 10.1016/j.jnucmat.2016.02.003

Behler J (2016) Perspective: Machine learning potentials for atomistic simulations. J Chem Phys 145:170901. doi: 10.1063/1.4966192

Béland LK, Anahory Y, Smeets D, et al (2013) Replenish and Relax: Explaining Logarithmic Annealing in Ion-Implanted \$c\$-Si. Phys Rev Lett 111:105502. doi: 10.1103/PhysRevLett.111.105502

Béland LK, Brommer P, El-Mellouhi F, et al (2011) Kinetic activation-relaxation technique. Phys Rev E 84:046704. doi: 10.1103/PhysRevE.84.046704

Béland LK, Osetsky YN, Stoller RE, Xu H (2015a) Kinetic Activation-Relaxation Technique and SelfEvolving Atomistic Kinetic Monte Carlo: Comparison of on-the-fly Kinetic Monte Carlo algorithms. Comput Mater Sci 100:124-134. doi: 10.1016/j.commatsci.2014.12.001

Béland LK, Osetsky YN, Stoller RE, Xu H (2015b) Interstitial loop transformations in FeCr. J Alloys Compd 640:219-225. doi: 10.1016/j.jallcom.2015.03.173

Bellon P, Averback RS (2015) Self-organization reactions under irradiation: Toward the design of radiation-resistant materials. pp 873-874

Bellon P, Enrique RA (2001) Interface stability and self-organization of precipitates under irradiation. Nucl Instrum Methods Phys Res Sect B Beam Interact Mater At 178:1-6. doi: 10.1016/S0168$583 \mathrm{X}(00) 00500-0$

Bertolus M, Freyss M, Dorado B, et al (2015) Linking atomic and mesoscopic scales for the modelling of the transport properties of uranium dioxide under irradiation. J Nucl Mater 462:475-495. doi: 10.1016/j.jnucmat.2015.02.026

Besco DG (1967) Computer Simulation of Point Defect Annealing in Metals

Bonny G, Castin N, Bakaev A, Terentyev D (2018) Kinetic Monte Carlo model for 1-D migration in a field of strong traps: Application to self-interstitial clusters in W-Re alloys. Comput Mater Sci 144:355-362. doi: 10.1016/j.commatsci.2017.12.024

Bonny G, Pasianot RC, Castin N, Malerba L (2009) Ternary Fe-Cu-Ni many-body potential to model reactor pressure vessel steels: First validation by simulated thermal annealing. Philos Mag 89:3531-3546. doi: 10.1080/14786430903299824 
Bonny G, Terentyev D, Bakaev A, et al (2013) On the thermal stability of late blooming phases in reactor pressure vessel steels: An atomistic study. J Nucl Mater 442:282-291. doi: 10.1016/j.jnucmat.2013.08.018

Bortz AB, Kalos MH, Lebowitz JL (1975) A new algorithm for Monte Carlo simulation of Ising spin systems. J Comput Phys 17:10-18. doi: 10.1016/0021-9991(75)90060-1

Bringa EM, Wirth BD, Caturla MJ, et al (2003) Metals far from equilibrium: From shocks to radiation damage. Nucl Instrum Methods Phys Res Sect B Beam Interact Mater At 202:56-63. doi: $10.1016 / \mathrm{S} 0168-583 \mathrm{X}(02) 01831-1$

Brommer P, Béland LK, Joly J-F, Mousseau N (2014) Understanding long-time vacancy aggregation in iron: A kinetic activation-relaxation technique study. Phys Rev B 90:134109. doi: 10.1103/PhysRevB.90.134109

Brommer P, Kiselev A, Schopf D, et al (2015) Classical interaction potentials for diverse materials from ab initio data: a review of potfit. Model Simul Mater Sci Eng 23:074002. doi: 10.1088/09650393/23/7/074002

Carpentier D, Jourdan T, Le Bouar Y, Marinica M-C (2017) Effect of saddle point anisotropy of point defects on their absorption by dislocations and cavities. Acta Mater 136:323-334. doi: 10.1016/j.actamat.2017.07.013

Castin N, Bonny G, Bakaev A, et al (2018) Object kinetic Monte Carlo model for neutron and ion irradiation in tungsten: Impact of transmutation and carbon impurities. J Nucl Mater 500:1525. doi: $10.1016 /$ j.jnucmat.2017.12.014

Castin N, Malerba L (2010) Calculation of proper energy barriers for atomistic kinetic Monte Carlo simulations on rigid lattice with chemical and strain field long-range effects using artificial neural networks. J Chem Phys 132:074507. doi: 10.1063/1.3298990

Castin N, Malerba L, Bonny G, et al (2009) Modelling radiation-induced phase changes in binary $\mathrm{FeCu}$ and ternary $\mathrm{FeCuNi}$ alloys using an artificial intelligence-based atomistic kinetic Monte Carlo approach. Nucl Instrum Methods Phys Res Sect B Beam Interact Mater At 267:3002-3008. doi: 10.1016/j.nimb.2009.06.092

Castin N, Pascuet MI, Malerba L (2011) Modeling the first stages of Cu precipitation in $\alpha$-Fe using a hybrid atomistic kinetic Monte Carlo approach. J Chem Phys 135:064502. doi: $10.1063 / 1.3622045$

Caturla MJ, Aliaga MJ, Martin-Bragado I, et al (2016) Microstructure Evolution in Fe and Fe-Cr Alloys with OKMC Methods. EPJ Web Conf 115:03001. doi: 10.1051/epjconf/201611503001

Caturla MJ, Diaz de la Rubia T, Fluss M (2003) Modeling microstructure evolution of f.c.c. metals under irradiation in the presence of He. J Nucl Mater 323:163-168. doi: 10.1016/j.jnucmat.2003.08.003

Caturla MJ, Diaz de la Rubia T, Victoria M, et al (2001) Multiscale modeling of radiation damage: applications to damage production by $\mathrm{GeV}$ proton irradiation of $\mathrm{Cu}$ and $\mathrm{W}$, and pulsed irradiation effects in $\mathrm{Cu}$ and Fe. J Nucl Mater 296:90-100. doi: 10.1016/S0022-3115(01)005694

Caturla MJ, Ortiz CJ, Fu CC (2008) Helium and point defect accumulation: (ii) kinetic modelling. Comptes Rendus Phys 9:401-408. doi: 10.1016/j.crhy.2007.09.004 
Caturla MJ, Soneda N, Alonso E, et al (2000a) Comparative study of radiation damage accumulation in $\mathrm{Cu}$ and Fe. J Nucl Mater 276:13-21. doi: 10.1016/S0022-3115(99)00220-2

Caturla MJ, Soneda N, Diaz de la Rubia T, Fluss M (2006) Kinetic Monte Carlo simulations applied to irradiated materials: The effect of cascade damage in defect nucleation and growth. J Nucl Mater 351:78-87. doi: 10.1016/j.jnucmat.2006.02.019

Caturla M-J, Wall M, Alonso E, et al (2000b) Heavy ion irradiation and annealing of lead: atomistic simulations and experimental validation. J Nucl Mater 276:186-193. doi: 10.1016/S00223115(99)00202-0

Chatterjee A, Vlachos DG (2007) An overview of spatial microscopic and accelerated kinetic Monte Carlo methods. J Comput-Aided Mater Des 14:253-308. doi: 10.1007/s10820-006-9042-9

Chiapetto M, Becquart CS, Domain C, Malerba L (2015) Nanostructure evolution under irradiation of $\mathrm{Fe}(\mathrm{C}) \mathrm{MnNi}$ model alloys for reactor pressure vessel steels. Nucl Instrum Methods Phys Res Sect B Beam Interact Mater At 352:56-60. doi: 10.1016/j.nimb.2014.11.102

Chiapetto M, Becquart CS, Malerba L (2016a) Simulation of nanostructural evolution under irradiation in Fe-9\%CrC alloys: An object kinetic Monte Carlo study of the effect of temperature and doserate. Nucl Mater Energy 9:565-570. doi: 10.1016/j.nme.2016.04.009

Chiapetto M, Malerba L, Puype A, Becquart CS (2016b) Object kinetic Monte Carlo study of the effect of grain boundaries in martensitic Fe-Cr-C alloys. Phys Status Solidi A 213:2981-2987. doi: $10.1002 /$ pssa.201600294

Chill ST, Henkelman G (2014) Molecular dynamics saddle search adaptive kinetic Monte Carlo. J Chem Phys 140:214110. doi: 10.1063/1.4880721

Choi YH, Joo HG (2013) Multiscale simulation of neutron induced damage in tritium breeding blankets with different spectral shifters. Fusion Eng Des 88:2471-2475. doi: 10.1016/j.fusengdes.2013.05.085

Dalla Torre J, Bocquet J-L, Doan NV, et al (2005) JERK, an event-based Kinetic Monte Carlo model to predict microstructure evolution of materials under irradiation. Philos Mag 85:549-558. doi: $10.1080 / 02678370412331320134$

De Backer A, Ortiz CJ, Domain C, et al (2013) Spatial effects in the $800 \mathrm{keV} 3 \mathrm{He}$ implantation in W followed by isochronal annealing at $900 \mathrm{~K}$. Nucl Instrum Methods Phys Res Sect B Beam Interact Mater At 303:87-90. doi: 10.1016/j.nimb.2012.10.025

De Backer A, Adjanor G, Domain C, et al (2015) Modeling of helium bubble nucleation and growth in austenitic stainless steels using an Object Kinetic Monte Carlo method. Nucl Instrum Methods Phys Res Sect B Beam Interact Mater At 352:107-114. doi: 10.1016/j.nimb.2014.11.110

Deo CS, Okuniewski MA, Srivilliputhur SG, et al (2007) Helium bubble nucleation in bcc iron studied by kinetic Monte Carlo simulations. J Nucl Mater 361:141-148. doi: 10.1016/j.jnucmat.2006.12.018

Diaz DLR, Soneda N, Caturla MJ, Alonso EA (1997) Defect production and annealing kinetics in elemental metals and semiconductors. J Nucl Mater 251:13-33

Djurabekova F, Malerba L, Pasianot RC, et al (2010) Kinetics versus thermodynamics in materials modeling: The case of the di-vacancy in iron. Philos Mag 90:2585-2595. doi: $10.1080 / 14786431003662515$ 
Djurabekova FG, Domingos R, Cerchiara G, et al (2007) Artificial intelligence applied to atomistic kinetic Monte Carlo simulations in Fe-Cu alloys. Nucl Instrum Methods Phys Res Sect B Beam Interact Mater At 255:8-12. doi: 10.1016/j.nimb.2006.11.039

Domain C, Becquart CS (2018) Solute - 〈111〉 interstitial loop interaction in $\alpha$-Fe: A DFT study. J Nucl Mater 499:582-594. doi: 10.1016/j.jnucmat.2017.10.070

Domain C, Becquart CS (2019) Object Kinetic Monte Carlo (OKMC): A Coarse-Grained Approach to Radiation Damage. In: Andreoni W, Yip S (eds) Handbook of Materials Modeling : Methods: Theory and Modeling. Springer International Publishing, Cham, pp 1-26

Domain C, Becquart CS, Malerba L (2004) Simulation of radiation damage in Fe alloys: an object kinetic Monte Carlo approach. J Nucl Mater 335:121-145. doi: 10.1016/j.jnucmat.2004.07.037

Domain C, Becquart CS, Van D (1999) Kinetic Monte Carlo simulations of FeCu alloys. Mater Res Soc Symp - Proc 538:217-222

Domingos RP, Cerchiara GM, Djurabekova F, Malerba L (2006) Artificial intelligence applied to simulation of radiation damage in ferritic alloys. In: Applied Artificial Intelligence. WORLD SCIENTIFIC, pp 883-890

Donev A, Bulatov VV, Oppelstrup T, et al (2010) A First-Passage Kinetic Monte Carlo algorithm for complex diffusion-reaction systems. J Comput Phys 229:3214-3236. doi: 10.1016/j.jcp.2009.12.038

Doran DG (1970) Computer simulation of displacement spike annealing. Radiat Eff 2:249-267. doi: $10.1080 / 00337576908243987$

Duan G, Li X, Xu Y, et al (2018) Clustering and segregation of small vacancy clusters near tungsten (0 0 1) surface. Nucl Instrum Methods Phys Res Sect B Beam Interact Mater At 414:29-37. doi: 10.1016/j.nimb.2017.10.007

Ejenstam J, Thuvander M, Olsson P, et al (2015) Microstructural stability of $\mathrm{Fe}-\mathrm{Cr}-\mathrm{Al}$ alloys at 450 550 ${ }^{\circ}$ C. J Nucl Mater 457:291-297. doi: 10.1016/j.jnucmat.2014.11.101

El-Mellouhi F, Mousseau N, Lewis LJ (2008) Kinetic activation-relaxation technique: An off-lattice self-learning kinetic Monte Carlo algorithm. Phys Rev B 78:153202. doi: 10.1103/PhysRevB.78.153202

Erhart P, Marian J (2011) Calculation of the substitutional fraction of ion-implanted He in an $\alpha$-Fe target. J Nucl Mater 414:426-430. doi: 10.1016/j.jnucmat.2011.05.017

Fichthorn KA, Weinberg WH (1991) Theoretical foundations of dynamical Monte Carlo simulations. J Chem Phys 95:1090. doi: 10.1063/1.461138

Fluss MJ, Wirth BD, Wall M, et al (2004) Temperature-dependent defect properties from ion-irradiation in $\mathrm{Pu}(\mathrm{Ga})$. J Alloys Compd 368:62-74. doi: 10.1016/j.jallcom.2003.08.080

Fujii T, Tohgo K, Kenmochi A, Shimamura Y (2015) Experimental and numerical investigation of stress corrosion cracking of sensitized type 304 stainless steel under high-temperature and high-purity water. Corros Sci 97:139-149. doi: 10.1016/j.corsci.2015.05.001

Galloway GJ, Ackland GJ (2013) Molecular dynamics and object kinetic Monte Carlo study of radiation-induced motion of voids and He bubbles in bcc iron. Phys Rev B 87:104106. doi: 10.1103/PhysRevB.87.104106 
Gámez B, Gámez L, Ortiz CJ, et al (2009) Object Kinetic Monte Carlo calculations of electron and He irradiation of nickel. J Nucl Mater 386-388:90-92. doi: 10.1016/j.jnucmat.2008.12.066

Gámez L, Gámez B, Caturla MJ, et al (2011) Object Kinetic Monte Carlo calculations of irradiated Fe$\mathrm{Cr}$ dilute alloys: The effect of the interaction radius between substitutional $\mathrm{Cr}$ and selfinterstitial Fe. Nucl Instrum Methods Phys Res Sect B Beam Interact Mater At 269:1684-1688. doi: 10.1016/j.nimb.2010.12.044

Gámez L, Martínez E, Perlado JM, et al (2007) Kinetic Monte Carlo modelling of neutron irradiation damage in iron. Fusion Eng Des 82:2666-2670. doi: 10.1016/j.fusengdes.2007.04.040

Gao F, Bacon DJ, Barashev AV, Heinisch HL (1999) Kinetic Monte Carlo annealing simulation of damage produced by cascades in alpha-iron. Mater Res Soc Symp - Proc 540:703-708

Ghoniem NM, Tong SH, Huang J, et al (2002) Mechanisms of dislocation-defect interactions in irradiated metals investigated by computer simulations. J Nucl Mater 307-311:843-851. doi: 10.1016/S0022-3115(02)01092-9

Gilbert MR, Sublet J-C (2011) Neutron-induced transmutation effects in W and W-alloys in a fusion environment. Nucl Fusion 51:. doi: 10.1088/0029-5515/51/4/043005

Guo X, Zhang X, Xue J, Li W (2013) KMC simulation of helium bubble formation in alpha-Fe. Nucl Instrum Methods Phys Res Sect B Beam Interact Mater At 307:77-80. doi: 10.1016/j.nimb.2012.12.108

Hehr BD (2014) Analysis of radiation effects in silicon using kinetic monte carlo methods. IEEE Trans Nucl Sci 61:2847-2854. doi: 10.1109/TNS.2014.2368075

Heinisch HL (1983) Defect production in simulated cascades: Cascade quenching and short-term annealing. J Nucl Mater 117:46-54. doi: 10.1016/0022-3115(83)90008-9

Heinisch HL, Singh BN, Golubov SI (2000) Kinetic Monte Carlo studies of the effects of Burgers vector changes on the reaction kinetics of one-dimensionally gliding interstitial clusters. J Nucl Mater 276:59-64. doi: 10.1016/S0022-3115(99)00169-5

Heinisch HL, Singh BN, Golubov SI (1999) Kinetic Monte Carlo study of mixed 1D/3D defect migration. J Comput-Aided Mater Des 6:277-282

Heinisch HL, Trinkaus H, Singh BN (2007) Kinetic Monte Carlo studies of the reaction kinetics of crystal defects that diffuse one-dimensionally with occasional transverse migration. J Nucl Mater 367-370:332-337. doi: 10.1016/j.jnucmat.2007.03.034

Heinisch HL, Weber WJ (2005) Computational model of alpha-decay damage accumulation in zircon. Nucl Instrum Methods Phys Res Sect B Beam Interact Mater At 228:293-298. doi: 10.1016/j.nimb.2004.10.059

Henkelman G, Jónsson H (2001) Long time scale kinetic Monte Carlo simulations without lattice approximation and predefined event table. J Chem Phys 115:9657. doi: 10.1063/1.1415500

Henkelman G, Jónsson H, Lelièvre T, et al (2018) Long-Timescale Simulations: Challenges, Pitfalls, Best Practices, for Development and Applications. In: Andreoni W, Yip S (eds) Handbook of Materials Modeling : Methods: Theory and Modeling. Springer International Publishing, Cham, pp 1-10 
Herbert FW, Krishnamoorthy A, Ma W, et al (2014) Dynamics of point defect formation, clustering and pit initiation on the pyrite surface. Electrochimica Acta 127:416-426. doi: 10.1016/j.electacta.2014.02.048

Hernández-Mayoral M, Gómez-Briceño D (2010) Transmission electron microscopy study on neutron irradiated pure iron and RPV model alloys. J Nucl Mater 399:146-153. doi: 10.1016/j.jnucmat.2009.11.013

Hobler G, Otto G (2003) Status and open problems in modeling of as-implanted damage in silicon. Mater Sci Semicond Process 6:1-14. doi: 10.1016/S1369-8001(03)00065-9

Hobler G, Otto G, Kovač D, et al (2005) Multiscale approach for the analysis of channeling profile measurements of ion implantation damage. Nucl Instrum Methods Phys Res Sect B Beam Interact Mater At 228:360-363. doi: 10.1016/j.nimb.2004.10.070

Hornbogen E, Glenn RC (1960) A Metallographic Study of Precipitation of Copper from Alpha Iron. Trans Met Soc AIME 218:1064

Hou J, Kong X-S, Li X-Y, et al (2016) Modification on theory of sink strength: An Object Kinetic Monte Carlo study. Comput Mater Sci 123:148-157. doi: 10.1016/j.commatsci.2016.06.024

Huang C-H, Gharaee L, Zhao Y, et al (2017) Mechanism of nucleation and incipient growth of Re clusters in irradiated W-Re alloys from kinetic Monte Carlo simulations. Phys Rev B 96:094108. doi: 10.1103/PhysRevB.96.094108

Hudson TS, Dudarev SL, Caturla M-J, Sutton AP (2005) Effects of elastic interactions on post-cascade radiation damage evolution in kinetic Monte Carlo simulations. Philos Mag 85:661-675. doi: 10.1080/14786430412331320026

Hudson TS, Dudarev SL, Sutton AP (2004) Suppression of interstitial cluster diffusion by oversized solute atoms. J Nucl Mater 329-333:971-976. doi: 10.1016/j.jnucmat.2004.04.066

Ito AM, Kato S, Takayama A, Nakamura H (2017) Automatic kinetic Monte-Carlo modeling for impurity atom diffusion in grain boundary structure of tungsten material. Nucl Mater Energy 12:353-360. doi: 10.1016/j.nme.2017.04.010

Jansson V, Malerba L, De Backer A, et al (2013) Sink strength calculations of dislocations and loops using OKMC. J Nucl Mater 442:218-226. doi: 10.1016/j.jnucmat.2013.08.052

Johnson MD, Caturla M-J, Díaz de la Rubia T (1998) A kinetic Monte-Carlo study of the effective diffusivity of the silicon self-interstitial in the presence of carbon and boron. J Appl Phys 84:1963-1967. doi: 10.1063/1.368328

Joly J-F, Béland LK, Brommer P, et al (2012) Optimization of the Kinetic Activation-Relaxation Technique, an off-lattice and self-learning kinetic Monte-Carlo method. J Phys Conf Ser 341:012007. doi: 10.1088/1742-6596/341/1/012007

Jourdan T, Crocombette J-P (2018) On the transfer of cascades from primary damage codes to rate equation cluster dynamics and its relation to experiments. Comput Mater Sci 145:235-243. doi: 10.1016/j.commatsci.2018.01.009

Jourdan T, Crocombette J-P (2012) Rate theory cluster dynamics simulations including spatial correlations within displacement cascades. Phys Rev B 86:054113. doi: 10.1103/PhysRevB.86.054113 
Jourdan T, Fu CC, Joly L, et al (2011) Direct simulation of resistivity recovery experiments in carbondoped $\alpha$-iron. Phys Scr 2011:014049. doi: 10.1088/0031-8949/2011/T145/014049

Jumel S, Domain C, Ruste J, et al (2002) Simulation of irradiation effects in reactor pressure vessel steels: The reactor for virtual experiments (REVE) project. J Test Eval 30:37-46

Kang HC, Weinberg WH (1989) Dynamic Monte Carlo with a proper energy barrier: Surface diffusion and two-dimensional domain ordering. J Chem Phys 90:2824. doi: 10.1063/1.455932

Khrushcheva O, Zhurkin EE, Malerba L, et al (2003) Copper precipitation in iron: a comparison between metropolis Monte Carlo and lattice kinetic Monte Carlo methods. Nucl Instrum Methods Phys Res Sect B Beam Interact Mater At 202:68-75. doi: 10.1016/S0168-583X(02)01830-X

Kittiratanawasin L, Smith R, Uberuaga BP, Sickafus KE (2009) Radiation damage and evolution of radiation-induced defects in Er 2 O 3 bixbyite. J Phys Condens Matter 21:115403. doi: $10.1088 / 0953-8984 / 21 / 11 / 115403$

Ko H, Deng J, Szlufarska I, Morgan D (2016) Ag diffusion in SiC high-energy grain boundaries: Kinetic Monte Carlo study with first-principle calculations. Comput Mater Sci 121:248-257. doi: 10.1016/j.commatsci.2016.04.027

Ko H, Szlufarska I, Morgan D (2017) Cs diffusion in SiC high-energy grain boundaries. J Appl Phys 122:105901. doi: 10.1063/1.4989389

Krasnochtchekov P, Averback RS, Bellon P (2007a) Precipitate stability and morphology in irradiation environments. JOM 59:46-50. doi: 10.1007/s11837-007-0054-Z

Krasnochtchekov P, Averback RS, Bellon P (2007b) Homogeneous phase separation in binary alloys under ion irradiation conditions: Role of interstitial atoms. Phys Rev B 75:144107. doi: 10.1103/PhysRevB.75.144107

Lanore J-M (1974) Simulation de l'evolution des defauts dans un reseau par le methode de monte-carlo. Radiat Eff 22:153-162. doi: 10.1080/10420157408230773

Lear CR, Bellon P, Averback RS (2017) Novel mechanism for order patterning in alloys driven by irradiation. Phys Rev B 96:104108. doi: 10.1103/PhysRevB.96.104108

Lee G-G, Kwon J, Kim DS (2009) A kinetic Monte Carlo approach for the analysis of trapping effect on the defect accumulation in neutron-irradiated Fe. Nucl Instrum Methods Phys Res Sect B Beam Interact Mater At 267:3214-3217. doi: 10.1016/j.nimb.2009.06.063

Li X, Liu W, Xu Y, et al (2016) Radiation resistance of nano-crystalline iron: Coupling of the fundamental segregation process and the annihilation of interstitials and vacancies near the grain boundaries. Acta Mater 109:115-127. doi: 10.1016/j.actamat.2016.02.028

Liu CL, Odette GR, Wirth BD, Lucas GE (1997a) A lattice Monte Carlo simulation of nanophase compositions and structures in irradiated pressure vessel Fe-Cu-Ni-Mn-Si steels. Mater Sci Eng A 238:202-209. doi: 10.1016/S0921-5093(97)00450-4

Mahmoud S, Trochet M, Restrepo OA, Mousseau N (2018) Study of point defects diffusion in nickel using kinetic activation-relaxation technique. Acta Mater 144:679-690. doi: 10.1016/j.actamat.2017.11.021

Maillard S, Martin G, Sabathier C (2016) Why a steady state void size distribution in irradiated UO2? A modeling approach. Nucl Instrum Methods Phys Res Sect B Beam Interact Mater At 374:5866. doi: 10.1016/j.nimb.2015.09.068 
Malek R, Mousseau N (2000) Dynamics of Lennard-Jones clusters: A characterization of the activationrelaxation technique. Phys Rev E 62:7723-7728. doi: 10.1103/PhysRevE.62.7723

Malerba L, Becquart CS, Domain C (2007) Object kinetic Monte Carlo study of sink strengths. J Nucl Mater 360:159-169. doi: 10.1016/j.jnucmat.2006.10.002

Marian J, Wirth BD, Perlado JM, et al (2001) Direct comparison between modeling and experiment: An $\alpha$-Fe ion implantation study. p R3.2.1-R3.2.6

Martin G, Bellon P (1996) Driven Alloys. In: Spaepen F, Ehrenreich H (eds) Solid State Physics. Academic Press, pp 189-331

Martin-Bragado I, Rivera A, Valles G, et al (2013) MMonCa: An Object Kinetic Monte Carlo simulator for damage irradiation evolution and defect diffusion. Comput Phys Commun 184:2703-2710. doi: $10.1016 /$ j.cpc.2013.07.011

Martínez E, Schwen D, Caro A (2015) Helium segregation to screw and edge dislocations in $\alpha$-iron and their yield strength. Acta Mater 84:208-214. doi: 10.1016/j.actamat.2014.10.066

Martínez E, Senninger O, Caro A, et al (2018) Role of Sink Density in Nonequilibrium Chemical Redistribution in Alloys. Phys Rev Lett 120:106101. doi: 10.1103/PhysRevLett.120.106101

Martinez-Martinez D, Herdes C, Vega LF (2018) Crystallization processes in bicomponent thin film depositions: Towards a realistic kinetic Monte Carlo simulation. Surf Coat Technol 343:38-48. doi: 10.1016/j.surfcoat.2017.11.022

McKay BD (1981) Practical graph isomorphism. Congr Numerantium 30:45-87

McKay BD, Piperno A (2014) Practical graph isomorphism, II. J Symb Comput 60:94-112. doi: 10.1016/j.jsc.2013.09.003

Méric de Bellefon G, Wirth BD (2011) Kinetic Monte Carlo (KMC) simulation of fission product silver transport through TRISO fuel particle. J Nucl Mater 413:122-131. doi: 10.1016/j.jnucmat.2011.04.010

Meslin E, Barbu A, Boulanger L, et al (2008) Cluster-dynamics modelling of defects in $\alpha$-iron under cascade damage conditions. J Nucl Mater 382:190-196. doi: 10.1016/j.jnucmat.2008.08.010

Meslin E, Lambrecht M, Hernández-Mayoral M, et al (2010) Characterization of neutron-irradiated ferritic model alloys and a RPV steel from combined APT, SANS, TEM and PAS analyses. J Nucl Mater 406:73-83. doi: 10.1016/j.jnucmat.2009.12.021

Messina L, Castin N, Domain C, Olsson P (2017) Introducing ab initio based neural networks for transition-rate prediction in kinetic Monte Carlo simulations. Phys Rev B 95:. doi: 10.1103/PhysRevB.95.064112

Messina L, Malerba L, Olsson P (2015) Stability and mobility of small vacancy-solute complexes in $\mathrm{Fe}-\mathrm{MnNi}$ and dilute Fe-X alloys: A kinetic Monte Carlo study. Nucl Instrum Methods Phys Res Sect B Beam Interact Mater At 352:61-66. doi: 10.1016/j.nimb.2014.12.032

Metropolis N, Rosenbluth AW, Rosenbluth MN, et al (1953) Equation of State Calculations by Fast Computing Machines. J Chem Phys 21:1087-1092. doi: 10.1063/1.1699114

Metropolis N, Ulam S (1949) The Monte Carlo Method. J Am Stat Assoc 44:335-341. doi: $10.1080 / 01621459.1949 .10483310$ 
Miller MK, Stoller RE, Russell KF (1996) Effect of neutron-irradiation on the spinodal decomposition of Fe-32\% Cr model alloy. J Nucl Mater 230:219-225. doi: 10.1016/0022-3115(96)80017-1

Monasterio PR, Wirth BD, Odette GR (2007) Kinetic Monte Carlo modeling of cascade aging and damage accumulation in $\mathrm{Fe}-\mathrm{Cu}$ alloys. $\mathrm{J}$ Nucl Mater 361:127-140. doi: 10.1016/j.jnucmat.2006.12.022

Morishita K, Sugano R, Wirth BD (2003) Thermal Stability of Helium-Vacancy Clusters and Bubble Formation - Multiscale Modeling Approach for Fusion Materials Development. Fusion Sci Technol 44:441-445. doi: 10.13182/FST03-A374

Nakashima K, Stoller RE, Xu H (2015) Recombination radius of a Frenkel pair and capture radius of a self-interstitial atom by vacancy clusters in bcc Fe. J Phys Condens Matter 27:335401. doi: $10.1088 / 0953-8984 / 27 / 33 / 335401$

Nandipati G, Setyawan W, Heinisch HL, et al (2015a) Displacement cascades and defect annealing in tungsten, Part II: Object kinetic Monte Carlo simulation of tungsten cascade aging. J Nucl Mater 462:338-344. doi: 10.1016/j.jnucmat.2014.09.067

Nandipati G, Setyawan W, Heinisch HL, et al (2015b) Displacement cascades and defect annealing in tungsten, Part III: The sensitivity of cascade annealing in tungsten to the values of kinetic parameters. J Nucl Mater 462:345-353. doi: 10.1016/j.jnucmat.2015.01.059

Ngayam-Happy R, Becquart CS, Domain C, Malerba L (2012) Formation and evolution of MnNi clusters in neutron irradiated dilute Fe alloys modelled by a first principle-based AKMC method. J Nucl Mater 426:198-207. doi: 10.1016/j.jnucmat.2012.03.033

Ngayam-Happy R, Olsson P, Becquart CS, Domain C (2010) Isochronal annealing of electron-irradiated dilute Fe alloys modelled by an ab initio based AKMC method: Influence of solute-interstitial cluster properties. J Nucl Mater 407:16-28. doi: 10.1016/j.jnucmat.2010.07.004

Nordlund K, Zinkle SJ, Sand AE, et al (2018) Primary radiation damage: A review of current understanding and models. J Nucl Mater 512:450-479. doi: 10.1016/j.jnucmat.2018.10.027

Norgett MJ, Robinson MT, Torrens IM (1975) A proposed method of calculating displacement dose rates. Nucl Eng Des 33:50-54. doi: 10.1016/0029-5493(75)90035-7

Oaks A, Yun D, Ye B, et al (2011) Kinetic Monte Carlo model of defect transport and irradiation effects in La-doped CeO2. J Nucl Mater 414:145-149. doi: 10.1016/j.jnucmat.2011.02.030

Oda T, Tanaka S (2011) Modeling of diffusivity of tritium interacting with F centers in Li2O. J Nucl Mater 417:743-747. doi: 10.1016/j.jnucmat.2010.12.132

Odette GR, Nanstad RK (2009) Predictive reactor pressure vessel steel irradiation embrittlement models: Issues and opportunities. JOM 61:17-23. doi: 10.1007/s11837-009-0097-4

Otto G, Kovač D, Hobler G (2005) Coupled BC/kLMC simulations of the temperature dependence of implant damage formation in silicon. Nucl Instrum Methods Phys Res Sect B Beam Interact Mater At 228:256-259. doi: 10.1016/j.nimb.2004.10.054

Oudriss A, Creus J, Bouhattate J, et al (2012) Grain size and grain-boundary effects on diffusion and trapping of hydrogen in pure nickel. Acta Mater 60:6814-6828. doi: 10.1016/j.actamat.2012.09.004

Pannier B (2017) Towards the prediction of microstructure evolution under irradiation of model ferritic alloys with an hybrid AKMC-OKMC approach. PhD dissertation, Université Lille 
Pareige C, Domain C, Olsson P (2009) Short- and long-range orders in Fe-Cr: A Monte Carlo study. J Appl Phys 106:104906. doi: 10.1063/1.3257232

Pareige C, Roussel M, Novy S, et al (2011) Kinetic study of phase transformation in a highly concentrated $\mathrm{Fe}-\mathrm{Cr}$ alloy: Monte Carlo simulation versus experiments. Acta Mater 59:24042411. doi: $10.1016 /$ j.actamat.2010.12.038

Pascuet MI, Castin N, Becquart CS, Malerba L (2011) Stability and mobility of Cu-vacancy clusters in $\mathrm{Fe}-\mathrm{Cu}$ alloys: A computational study based on the use of artificial neural networks for energy barrier calculations. J Nucl Mater 412:106-115. doi: 10.1016/j.jnucmat.2011.02.038

Payet A, Luce FP, Curfs C, et al (2016) Damage accumulation during cryogenic and room temperature implantations in strained SiGe alloys. Mater Sci Semicond Process 42:247-250. doi: 10.1016/j.mssp.2015.07.059

Pedersen A, Jónsson H (2009) Simulations of hydrogen diffusion at grain boundaries in aluminum. Acta Mater 57:4036-4045. doi: 10.1016/j.actamat.2009.04.057

Pelaz L, Marqués LA, López P, et al (2007) Multiscale modeling of radiation damage and annealing in Si. Nucl Instrum Methods Phys Res Sect B Beam Interact Mater At 255:95-100. doi: 10.1016/j.nimb.2006.11.035

Perez D, Sandoval L, Blondel S, et al (2017) The mobility of small vacancy/helium complexes in tungsten and its impact on retention in fusion-relevant conditions. Sci Rep 7:2522. doi: $10.1038 / \mathrm{s} 41598-017-02428-2$

Perlado JM, Lodi D, Marian J, et al (2003) Time-Dependent Neutronics in Structural Materials of Inertial Fusion Reactors and Simulation of Defect Accumulation in Pulsed Fe and SiC. Fusion Sci Technol 43:384-392. doi: 10.13182/FST03-A282

Perlado JM, Malerba L, de la R (1998) MD simulation of high energy cascades and damage accumulation in $\beta-\mathrm{SiC}$ in inertial fusion conditions. Fusion Technol 34:840-847

Ramasubramaniam A, Itakura M, Ortiz M, Carter EA (2008) Effect of atomic scale plasticity on hydrogen diffusion in iron: Quantum mechanically informed and on-the-fly kinetic Monte Carlo simulations. J Mater Res 23:2757-2773. doi: 10.1557/JMR.2008.0340

Restrepo OA, Mousseau N, Trochet M, et al (2018) Carbon diffusion paths and segregation at highangle tilt grain boundaries in lensuremath $\{\backslash$ alpha $\}$-Fe studied by using a kinetic activationrelation technique. Phys Rev B 97:054309. doi: 10.1103/PhysRevB.97.054309

Rivera A, Valles G, Caturla MJ, Martin-Bragado I (2013) Effect of ion flux on helium retention in helium-irradiated tungsten. Nucl Instrum Methods Phys Res Sect B Beam Interact Mater At 303:81-83. doi: 10.1016/j.nimb.2012.10.038

Robinson MT (1989) Slowing-down time of energetic atoms in solids. Phys Rev B 40:10717-10726. doi: 10.1103/PhysRevB.40.10717

Rottler J, Srolovitz DJ, Car R (2007) Kinetic Monte Carlo study of radiation-induced segregation in model metallic alloys. Philos Mag 87:3945-3958. doi: 10.1080/14786430701429904

Samolyuk GD, Barashev AV, Golubov SI, et al (2014) Analysis of the anisotropy of point defect diffusion in hcp Zr. Acta Mater 78:173-180. doi: 10.1016/j.actamat.2014.06.024

Sanchez JM, Ducastelle F, Gratias D (1984) Generalized cluster description of multicomponent systems. Phys Stat Mech Its Appl 128:334-350. doi: 10.1016/0378-4371(84)90096-7 
Schmauder S, Binkele P (2002) Atomistic computer simulation of the formation of Cu-precipitates in steels. Comput Mater Sci 24:42-53. doi: 10.1016/S0927-0256(02)00163-5

Sharafat S, Takahashi A, Nagasawa K, Ghoniem N (2009) A description of stress driven bubble growth of helium implanted tungsten. J Nucl Mater 389:203-212. doi: 10.1016/j.jnucmat.2009.02.027

Sivak AB, Sivak PA, Romanov VA, Chernov VM (2015) Effect of external stresses on efficiency of dislocation sinks in $\mathrm{BCC}(\mathrm{Fe}, \mathrm{V})$ and $\mathrm{FCC}(\mathrm{Cu})$ crystals. Inorg Mater Appl Res 6:466-472. doi: $10.1134 / \mathrm{S} 2075113315050184$

Soisson F (2006) Kinetic Monte Carlo simulations of radiation induced segregation and precipitation. J Nucl Mater 349:235-250. doi: 10.1016/j.jnucmat.2005.11.003

Soisson F, Barbu A, Martin G (1996a) Monte Carlo simulations of copper precipitation in dilute ironcopper alloys during thermal ageing and under electron irradiation. Acta Mater 44:3789-3800. doi: $10.1016 / 1359-6454(95) 00447-5$

Soisson F, Becquart CS, Castin N, et al (2010) Atomistic Kinetic Monte Carlo studies of microchemical evolutions driven by diffusion processes under irradiation. J Nucl Mater 406:55-67. doi: 10.1016/j.jnucmat.2010.05.018

Soisson F, Fu C-C (2007) Cu-precipitation kinetics in $\alpha$ - Fe from atomistic simulations: Vacancytrapping effects and Cu-cluster mobility. Phys Rev B 76:214102. doi: 10.1103/PhysRevB.76.214102

Soisson F, Jourdan T (2016) Radiation-accelerated precipitation in Fe-Cr alloys. Acta Mater 103:870881. doi: $10.1016 /$ j.actamat.2015.11.001

Soisson F, Meslin E, Tissot O (2018) Atomistic modeling of $\alpha$ ' precipitation in $\mathrm{Fe}-\mathrm{Cr}$ alloys under charged particles and neutron irradiations: Effects of ballistic mixing and sink densities. J Nucl Mater 508:583-594. doi: 10.1016/j.jnucmat.2018.06.015

Soneda N, Ishino S, Takahashi A, Dohi K (2003) Modeling the microstructural evolution in bcc-Fe during irradiation using kinetic Monte Carlo computer simulation. J Nucl Mater 323:169-180. doi: 10.1016/j.jnucmat.2003.08.021

Soneda N, Rubia TD de la (1998) Defect production, annealing kinetics and damage evolution in $\alpha$-Fe: An atomic-scale computer simulation. Philos Mag A 78:995-1019. doi: $10.1080 / 01418619808239970$

Souidi A, Becquart CS, Domain C, et al (2006) Dependence of radiation damage accumulation in iron on underlying models of displacement cascades and subsequent defect migration. J Nucl Mater 355:89-103. doi: 10.1016/j.jnucmat.2006.04.009

Souidi A, Hou M, Becquart CS, et al (2011) On the correlation between primary damage and long-term nanostructural evolution in iron under irradiation. J Nucl Mater 419:122-133. doi: 10.1016/j.jnucmat.2011.08.049

Stoller RE, Golubov SI, Domain C, Becquart CS (2008) Mean field rate theory and object kinetic Monte Carlo: A comparison of kinetic models. J Nucl Mater 382:77-90. doi: 10.1016/j.jnucmat.2008.08.047

Suzudo T, Golubov SI, Stoller RE, et al (2012) Annealing simulation of cascade damage in $\alpha$-Fe Damage energy and temperature dependence analyses. J Nucl Mater 423:40-46. doi: 10.1016/j.jnucmat.2012.01.014 
Suzudo T, Yamaguchi M, Hasegawa A (2015) Migration of rhenium and osmium interstitials in tungsten. J Nucl Mater 467:418-423. doi: 10.1016/j.jnucmat.2015.05.051

Suzudo T, Yamaguchi M, Kabraki H, Ebihara K-I (2010) Multiscale modeling of helium-vacancy cluster nucleation under irradiation: A kinetic Monte-Carlo approach. pp 29-35

Swinburne TD, Marinica M-C (2018) Unsupervised Calculation of Free Energy Barriers in Large Crystalline Systems. Phys Rev Lett 120:135503. doi: 10.1103/PhysRevLett.120.135503

Terentyev D, Martin-Bragado I (2015) Evolution of dislocation loops in iron under irradiation: The impact of carbon. Scr Mater 97:5-8. doi: 10.1016/j.scriptamat.2014.10.021

Trochet M, Béland LK, Joly J-F, et al (2015) Diffusion of point defects in crystalline silicon using the kinetic activation-relaxation technique method. Phys Rev B 91:224106. doi: 10.1103/PhysRevB.91.224106

Trushin O, Karim A, Kara A, Rahman TS (2005) Self-learning kinetic Monte Carlo method: Application to $\mathrm{Cu}(111)$. Phys Rev B 72:115401. doi: 10.1103/PhysRevB.72.115401

Uberuaga BP, Andersson DA (2015) Uranium vacancy mobility at the $\Sigma 5$ symmetric tilt and $\Sigma 5$ twist grain boundaries in UO2. Comput Mater Sci 108:80-87. doi: 10.1016/j.commatsci.2015.06.017

Uberuaga BP, Perez D (2018) Computational Methods for Long-Timescale Atomistic Simulations. In: Andreoni W, Yip S (eds) Handbook of Materials Modeling: Methods: Theory and Modeling. Springer International Publishing, Cham, pp 1-6

Valles G, L. Cazalilla A, Gonzalez C, et al (2015) A multiscale approach to defect evolution in tungsten under helium irradiation. Nucl Instrum Methods Phys Res Sect B Beam Interact Mater At 352:100-103. doi: 10.1016/j.nimb.2014.12.034

Valles G, Martin-Bragado I, Nordlund K, et al (2017a) Temperature dependence of underdense nanostructure formation in tungsten under helium irradiation. J Nucl Mater 490:108-114. doi: 10.1016/j.jnucmat.2017.04.021

Valles G, Panizo-Laiz M, González C, et al (2017b) Influence of grain boundaries on the radiationinduced defects and hydrogen in nanostructured and coarse-grained tungsten. Acta Mater 122:277-286. doi: 10.1016/j.actamat.2016.10.007

Van der Ven A, Ceder G, Asta M, Tepesch PD (2001) First-principles theory of ionic diffusion with nondilute carriers. Phys Rev B 64.. doi: 10.1103/PhysRevB.64.184307

Vattré A, Jourdan T, Ding H, et al (2016) Non-random walk diffusion enhances the sink strength of semicoherent interfaces. Nat Commun 7:10424. doi: 10.1038/ncomms10424

Vincent E, Becquart CS, Domain C (2006) Solute interaction with point defects in $\alpha$ Fe during thermal ageing: A combined ab initio and atomic kinetic Monte Carlo approach. J Nucl Mater 351:8899. doi: 10.1016/j.jnucmat.2006.02.018

Vincent E, Becquart CS, Domain C (2008a) Microstructural evolution under high flux irradiation of dilute $\mathrm{Fe}-\mathrm{CuNiMnSi}$ alloys studied by an atomic kinetic Monte Carlo model accounting for both vacancies and self interstitials. J Nucl Mater 382:154-159. doi: 10.1016/j.jnucmat.2008.08.019

Vincent E, Becquart CS, Pareige C, et al (2008b) Precipitation of the $\mathrm{FeCu}$ system: A critical review of atomic kinetic Monte Carlo simulations. J Nucl Mater 373:387-401. doi: 10.1016/j.jnucmat.2007.06.016 
Vineyard GH (1957) Frequency factors and isotope effects in solid state rate processes. J Phys Chem Solids 3:121-127. doi: 10.1016/0022-3697(57)90059-8

Voter AF, Montalenti F, Germann TC (2002) Extending the Time Scale in Atomistic Simulation of Materials. Annu Rev Mater Res 32:321-346. doi: 10.1146/annurev.matsci.32.112601.141541

$\mathrm{Vu}$ THY, Ramjauny Y, Hayoun M, et al (2015) On the evolution of the steady state in gold-silica nanocomposites under sustained irradiation. J Appl Phys 117:174305. doi: 10.1063/1.4919019

Vu THY, Ramjauny Y, Rizza G, Hayoun M (2016) Effect of the size of nanoparticles on their dissolution within metal-glass nanocomposites under sustained irradiation. J Appl Phys 119:034302. doi: $10.1063 / 1.4939974$

Wallenius J, Olsson P, Malerba L, Terentyev D (2007) Simulation of thermal ageing and radiation damage in Fe-Cr. Nucl Instrum Methods Phys Res Sect B Beam Interact Mater At 255:68-74. doi: 10.1016/j.nimb.2006.11.063

Wang Y, Yin J, Liu X, et al (2017) Precipitation kinetics in binary $\mathrm{Fe}-\mathrm{Cu}$ and ternary $\mathrm{Fe}-\mathrm{Cu}-\mathrm{Ni}$ alloys via kMC method. Prog Nat Sci Mater Int 27:460-466. doi: 10.1016/j.pnsc.2017.06.005

Warrier M, Bhardwaj U, Bukkuru S (2016) Multi-scale Modeling of Radiation Damage: Large Scale Data Analysis. J Phys Conf Ser 759:012078. doi: 10.1088/1742-6596/759/1/012078

Wen M, Ghoniem NM, Singh BN (2005) Dislocation decoration and raft formation in irradiated materials. Philos Mag 85:2561-2580. doi: 10.1080/14786430500154281

Wen M, Takahashi A, Ghoniem NM (2009) Kinetics of self-interstitial cluster aggregation near dislocations and their influence on hardening. J Nucl Mater 392:386-395. doi: 10.1016/j.jnucmat.2008.10.029

Wirth BD, Bringa EM (2004) A kinetic monte carlo model for helium diffusion and clustering in fusion environments. pp 80-84

Wirth BD, Caturla MJ, Diaz de la Rubia T, et al (2001) Mechanical property degradation in irradiated materials: A multiscale modeling approach. Nucl Instrum Methods Phys Res Sect B Beam Interact Mater At 180:23-31. doi: 10.1016/S0168-583X(01)00392-5

Wirth BD, Odette GR (1998) Kinetic Lattice Monte Carlo simulations of diffusion and decomposition kinetics in $\mathrm{Fe}-\mathrm{Cu}$ alloys: Embedded atom and nearest neighbor potentials. pp 151-155

Wood WW, Parker FR (1957) Monte Carlo Equation of State of Molecules Interacting with the LennardJones Potential. I. A Supercritical Isotherm at about Twice the Critical Temperature. J Chem Phys 27:720-733. doi: 10.1063/1.1743822

Wu B, Li S, Zhang Y (2015) Optimizing Parallel Kinetic Monte Carlo Simulation by Communication Aggregation and Scheduling. In: Big Data Technology and Applications. Springer, Singapore, pp 282-297

Wu G-Y, Hu N-W, Deng H-Q, et al (2017) Simulation of radiation damages in molybdenum by combining molecular dynamics and OKMC. Nucl Sci Tech 28:3. doi: 10.1007/s41365-0160164-9

Xiao W-J, Wu G-Y, Li M-H, et al (2016) MD and OKMC simulations of the displacement cascades in nickel. Nucl Sci Tech 27:57. doi: 10.1007/s41365-016-0057-y 
Xu D, Wirth BD, Li M, Kirk MA (2012a) Defect microstructural evolution in ion irradiated metallic nanofoils: Kinetic Monte Carlo simulation versus cluster dynamics modeling and in situ transmission electron microscopy experiments. Appl Phys Lett 101:101905. doi: $10.1063 / 1.4748980$

Xu H, Osetsky YN, Stoller RE (2011) Simulating complex atomistic processes: On-the-fly kinetic Monte Carlo scheme with selective active volumes. Phys Rev B 84:. doi: 10.1103/PhysRevB.84.132103

Xu H, Osetsky YN, Stoller RE (2012b) Cascade annealing simulations of bcc iron using object kinetic Monte Carlo. J Nucl Mater 423:102-109. doi: 10.1016/j.jnucmat.2012.01.020

Xu H, Stoller RE, Osetsky YN, Terentyev D (2013) Solving the Puzzle of \$ $\langle 100\rangle$ Interstitial Loop Formation in bcc Iron. Phys Rev Lett 110:265503. doi: 10.1103/PhysRevLett.110.265503

Yang X, Hassanein A (2014) Kinetic Monte Carlo simulation of hydrogen diffusion on tungsten reconstructed (001) surface. Fusion Eng Des 89:2545-2549. doi: 10.1016/j.fusengdes.2014.06.001

Yang X, Oyeniyi WO (2017) Kinetic Monte Carlo simulation of hydrogen diffusion in tungsten. Fusion Eng Des 114:113-117. doi: 10.1016/j.fusengdes.2016.12.012

Yang Z, Blondel S, Hammond KD, Wirth BD (2017) Kinetic Monte Carlo Simulations of Helium Cluster Nucleation in Tungsten with Preexisting Vacancies. Fusion Sci Technol 71:60-74. doi: 10.13182/FST16-111

Ye J, Bellon P (2004) Nanoscale patterning of chemical order induced by displacement cascades in irradiated alloys. I. A kinetic Monte Carlo study. Phys Rev B 70:094104. doi: 10.1103/PhysRevB.70.094104

Yi X, Jenkins ML, Hattar K, et al (2015) Characterisation of radiation damage in W and W-based alloys from $2 \mathrm{MeV}$ self-ion near-bulk implantations. Acta Mater 92:163-177. doi: 10.1016/j.actamat.2015.04.015

Yoshiie T, Ito T, Iwase H, et al (2011) Multi-scale modeling of irradiation effects in spallation neutron source materials. Nucl Instrum Methods Phys Res Sect B Beam Interact Mater At 269:1740 1743. doi: 10.1016/j.nimb.2010.11.076

Young WM, Elcock EW (1966) Monte Carlo studies of vacancy migration in binary ordered alloys: I. Proc Phys Soc 89:735. doi: 10.1088/0370-1328/89/3/329

Zhang X, Beach JA, Wang M, et al (2016) Precipitation kinetics of dilute $\mathrm{Cu}-\mathrm{W}$ alloys during lowtemperature ion irradiation. Acta Mater 120:46-55. doi: 10.1016/j.actamat.2016.08.043

Zhang X, Shu S, Bellon P, Averback RS (2015) Precipitate stability in $\mathrm{Cu}-\mathrm{Ag}-\mathrm{W}$ system under hightemperature irradiation. Acta Mater 97:348-356. doi: 10.1016/j.actamat.2015.06.045

Ziegler JF, Ziegler MD, Biersack JP (2010) SRIM - The stopping and range of ions in matter (2010). Nucl Instrum Methods Phys Res Sect B Beam Interact Mater At 268:1818-1823. doi: 10.1016/j.nimb.2010.02.091 